\title{
COMPLEXITY REDUCTION FOR CALIBRATION TO AMERICAN OPTIONS
}

\author{
OLENA BURKOVSKA, KATHRIN GLAU, MIRCO MAHLSTEDT, AND BARBARA WOHLMUTH
}

\begin{abstract}
American put options are among the most frequently traded single stock options, and their calibration is computationally challenging since no closed-form expression is available. Due to the higher flexibility in comparison to European options, the mathematical model involves additional constraints, and a variational inequality is obtained. We use the Heston stochastic volatility model to describe the price of a single stock option. In order to speed up the calibration process, we apply two model reduction strategies. Firstly, we introduce a reduced basis method. We thus reduce the computational complexity of solving the parametric PDE drastically, compared to a classical finite element method, which renders applications of standard minimization algorithms for the calibration significantly faster. Secondly, we apply the so-called de-Americanization strategy. Here, the main idea is to reformulate the calibration problem for American options as a problem for European options and to exploit closed-form solutions. Both reduction techniques are systematically compared and tested for both synthetic and market data sets.
\end{abstract}

\section{INTRODUCTION}

Automatic and high-speed trading give rise to new computational challenges in the calibration of financial models. Both the frequent tradings and the constantly changing market situations make a steady recalibration necessary. Reliable risk assessment requires the estimation of the model parameters with adequate speed and precision.

In calibration procedures, the model parameters are chosen to optimally fit the option prices of the most liquid products. The calibration problem can be formulated as a least-squares minimization problem, namely to find a model parameter constellation that minimizes the quadratic discrepancy between the model and market option prices. These problems typically require the computation of model prices many times for different values of the parameters, which incurs to a high computational cost, in proportion with the effort of the underlying pricing routine.

Considering index derivatives, the most liquid options typically are of European style and the major part of the literature focuses on calibration to this class of options. Moreover, the availability of fast and highly accurate prices of European options for the purpose of calibration ranges among the basic model requirement. The most frequently traded single stock options, however, are of American style, which makes it necessary to use them for calibration as well. American option prices are path-dependent, as the option holder has the right to exercise at any time until maturity. This makes the design of methods to price options of American style considerably more involved than those for their European counterparts.

Key words and phrases. Reduced basis method, model reduction, American option, calibration, Heston model, de-Americanization.

This work was partly supported by: DFG grant WO671/11-1; International Research Training Group IGDK1754, funded by the German Research Foundation (DFG) and the Austrian research fund (FWF); KPMG Center of Excellence in Risk Management. 
Both European and American options can be priced by solving a parameter-dependent partial differential equation (PDE) in time and asset price, see, e.g., Achdou and Pironneau (2005a); Hilber et al. (2013) and the references therein. However, due to the higher flexibility of exercising American options compared to European options, the pricing PDE for an American options has to be enriched by a suitable inequality constraint, reflecting the early-exercise feature. The problem under consideration can be reformulated as a weak variational inequality problem to which semi-smooth Newton schemes can be applied as non-linear solvers. Classical discretization schemes such as finite elements or finite differences then require rather high-dimensional basis spaces, which leads to large systems to be solved.

To tackle the rising computational challenges, numerical techniques to reduce the complexity of the pricing problem are especially promising. Therefore, in order to accelerate the calibration to American options while still providing accurate results, we explore two different types of complexity reduction techniques. The first is based on reduced basis methods (RBM), which are tailored to efficiently solve parameter-dependent partial differential equations. In contrast, the second, de-Americanization method (DAS), exploits the fact that semi-closed form solutions can be accessed for the structurally simpler European options.

The key idea of the reduced basis method is to replace the basis of the standard finite element method (FEM) by a basis that is adapted to the parametric problem. This is achieved by forming the basis functions as linear combinations of suitable snapshot solutions, i.e. solutions of the PDE with specific choices of parameters. The striking advantage of this approach is that it typically is able to generate a basis of very small dimension compared to the classical basis functions, such as the local hat functions employed in FEM, that leads to the same range of accuracy. Thus, we obtain dense algebraic systems of remarkably smaller size. This leads to an iterative approximation procedure, which is considerably faster to solve when compared to the classical FEM. For this reason, RBM have been extensively studied and successfully applied in different fields of numerical analysis and engineering sciences over the last decade, see, e.g., Quarteroni et al. (2016); Hesthaven et al. (2016) and the references therein. First and highly promising results of RBM for option pricing can be found in Cont et al. (2011); Pironneau (2011, 2012). Recent work on model reduction techniques in finance with a primary focus on proper orthogonal decomposition (POD) methods include, e.g., Sachs and Schneider (2014); Pironneau (2012); Peherstorfer et al. (2015), and RBM, e.g., Mayerhofer and Urban (2016). Moreover, the RBM has been applied to accelerate the model calibration to European options, see Cont et al. (2011); Pironneau (2009). This motivates us to extend the approach to the more involved case of American options, which to the best of our knowledge, has not yet been addressed.

To this aim, we apply the RBM to price American options in the calibration routine and we investigate the performance of the method. American option prices, as described above, can be described by parabolic variational inequalites. The construction of an appropriate reduced basis space is much more challenging than for variational equalities. To tackle this, POD-Angle-Greedy strategies Burkovska et al. (2015) or non-negative matrix factorization algorithms Balajewicz et al. (2016); Balajewicz and Toivanen (2016) can be used. We also mention the relevant works on RBM for variational inequalities for the stationary case Haasdonk et al. (2012); Zhang et al. (2016) and instationary case in a space-time framework, e.g., Glas and Urban (2014). Here, we rely on the RBM to price American options, which has been developed in Burkovska et al. (2015); Burkovska (2016); Balajewicz and Toivanen (2016). 
Whereas discretization techniques for PDEs, such as FEM or RBM, form a rather flexible and abstract framework that have proven highly beneficial in different fields of applications, the de-Americanization technique is specially designed for the valuation of American options. It has been developed in the financial industry, where it is considered to be the benchmark method, see Carr and Wu (2010); Burkovska et al. (2018). This technique first transforms the price into a pseudo-European option price and secondly calibrates the European option by directly applying the computationally less expensive closed-form solution. In Burkovska et al. (2018), the de-Americanization technique is studied numerically, which reveals its strengths as well as limitations.

In this article, we investigate both model reduction techniques (RBM and de-Americanization) numerically for calibration of the Heston model, and we compare their performances.

The rest of the paper is structured as follows: We conclude the introduction with a short discussion of related literature. Then we briefly introduce the Heston model and the calibration procedure in Section 2. In Section 3 and Section 4 we discuss different pricing methodologies considered in this paper. We outline the main idea of the de-Americanization method in Section 3. The reduced basis method we present in Section 4. Here, we derive the variational formulation of the problem, present its discretized counterpart, and construct the reduced order model. Section 5 is devoted to the description of the calibration procedure based on the example of the Heston model. In Section 6 we present numerical results and comparative study of the calibration procedure with the reduced basis and the deAmericanization methods for synthetic as well as for marked data sets.

1.1. Related literature. The calibration problem can be also studied in the context of the theory of inverse problems and we provide a brief overview of the related literature. Many works are concerned with reconstructing the volatility surface for European options in the local-volatility variant of the Black-Scholes model. This then leads to an infinitedimensional inverse problem, where a distributed parameter needs to be reconstructed from the given market data. We refer, e.g., to Bouchouev and Isakov (1997), where unique solvability and stability of the inverse problem are analyzed, and to Egger and Engl (2005), where an appropriate Tikhonov regularization strategy is proposed to address the inherent ill-posedness of the problem. Similarly, in Achdou (2005); Achdou and Pironneau (2005b), a Tikhonov regularized problem with a local volatility is considered for American options, and the existence of solutions is derived together with their optimality conditions. In the present case, we consider the stochastic volatility Heston model. In the resulting inverse problem, we are confronted with the task of recovering only a finite number of global parameters. Since the dimension of the parameter space is typically much smaller then the number of available market observations, the inverse problem is over-determined. Therefore, we set up a least-squares minimization problem without including a regularization term.

We note that for calibration one can also work directly in the stochastic framework and compute the model prices, e.g., using a Monte-Carlo method by applying a backward regression scheme as in Longstaff and Schwartz (2001) or by using different Monte-Carlo estimates as in e.g. Broadie and Glasserman (1997); Fu et al. (2001); Rogers (2002). Alternatively one can apply (binomial) tree methods as e.g. in Cox et al. (1979); Rubinstein (1994). For European options, closed-form solutions can be used, or FFT techniques, see, e.g., Mrázek et al. (2014); Schoutens et al. (2004); Gerlich et al. (2012). Fourier transform based pricing methods have been extended to price American options, see for instance Fang and Oosterlee (2011) and Levendorskii (2004). These methods are applicable when the Fourier transform of the modelling stochastic process evaluated at fixed times is available 
in closed-form. In this article we focus on a PDE-based approach that has a more general scope.

\section{Problem formulation}

To calculate the price of an option (European or American) in a specific model, suitable data must be provided. These data consist of the current asset price $S_{0}>0$, the maturity time $T>0$, the strike price $K>0$, and the set of model parameters, such as, e.g., the interest rate, the long-run variance, the rate of mean reversion, the volatility of volatility and the correlation in the Heston model. We denote by the vector $\boldsymbol{\mu} \in \mathcal{P}$ the set of parameters, where $\mathcal{P} \subset \mathbb{R}^{p}$ is a parameter domain. Whereas the first three components, $S_{0}, K, T$, are known and provided by the market data, the input parameter vector $\boldsymbol{\mu}$ is not known a priori, except for the interest rate $r>0$, and needs to be estimated from the market. These types of problems are referred to as parameter identification or calibration problems. That is, given a set of observations of market option prices, we are interested in the parameter $\boldsymbol{\mu}$ that provides the best fit to the observed market data.

Generally, the market data is characterized by the actual spot price $S_{0}$ and the market option prices $P_{i}^{\text {obs }}=P^{\text {obs }}\left(S_{0}, T_{i}, K_{i}\right)$ for different maturities $T_{i}$ and for different strikes $K_{i}$, $i=1, \ldots, M$. Mathematically, the calibration of option prices can be stated as a least squares minimization problem: find $\boldsymbol{\mu} \in \mathcal{P}$, that solves

$$
\min _{\boldsymbol{\mu}} J(\boldsymbol{\mu}), \quad J(\boldsymbol{\mu}):=\frac{1}{M} \sum_{i=1}^{M}\left|P_{i}^{\mathrm{obs}}-P_{i}(\boldsymbol{\mu})\right|^{2},
$$

where $P_{i}(\boldsymbol{\mu})=P\left(S_{0}, T_{i}, K_{i} ; \boldsymbol{\mu}\right)$ are the model prices. Numerous approaches exist to calculate the model price $P(\boldsymbol{\mu})$. The most common ones are analytic formulae, Monte Carlo methods, binomial/trinomial trees, numerical method for approximating the PDEs (finite elements, finite differences). While the Monte Carlo method is the most general approach that is applicable to many models, it suffers from the expensive computational cost and lack of accuracy. In contrast, the numerical approximation of the PDEs are more accurate methods, however they also come with the high computational cost as soon as the number of degrees of freedom of the discrete system increases. In the context of the calibration problems, where multiple evaluation of the solution for different parameter values are required, the computational cost is then significantly amplified. To make these problems feasible in application, approaches with a low computational cost are in favor.

In the following, we investigate two different computationally efficient approaches which also preserve the accuracy of problem, the RBM, described in Section 4.5 and DAS, presented in Section 3. For the numerical experiments of this paper we choose the Heston model, Gerlich et al. (2012); Mrázek et al. (2014); Schoutens et al. (2004), and consider the pricing of American and European put options. We point out that the methodologies presented here are applicable more generally to other models and option types as well.

2.1. Heston model. The Heston model, considered as a benchmark example in this paper, is described by the following stock price (2.2a) and volatility (2.2b) dynamics,

$$
\begin{aligned}
& d S=\iota S d \tau+\sigma S d W^{1}, \\
& d \nu=\kappa(\gamma-\nu) d \tau+\xi \sqrt{\nu} d W^{2} .
\end{aligned}
$$

The asset price $S:=\left\{S_{\tau}: \tau \geq 0\right\}$ exhibits geometric Brownian motion with Wiener process $W^{1}$, drift $\iota$ and volatility $\sigma:=\sqrt{\nu}$. The stochastic instantaneous variance $\nu:=\left\{\nu_{\tau}: \tau \geq 0\right\}$ is driven by a mean-reverting square-root process (known as the Cox-Ingersoll-Ross (CIR) 
process) with long-run variance $\gamma>0$, rate of mean reversion $\kappa>0$, and volatility of variance (also called the volatility of volatility) $\xi>0$. The Wiener processes $W^{1}$ and $W^{2}$ are correlated by $\rho \in[-1,1]$. Moreover, the so-called Feller condition is assumed, which states that the variance process $(2.2 \mathrm{~b})$ is strictly positive if the parameters satisfy

$$
2 \kappa \gamma>\xi^{2}
$$

see, e.g., Janek et al. (2011). Unless otherwise stated, we focus on setting the parameters such that the Feller condition is always fulfilled.

\section{De-Americanization Strategy (DAS)}

The de-Americanization method transforms the observed American market prices into pseudo-European prices prior to the calibration. The rationale behind the de-Americanization strategy is the following. Calibrating a sophisticated model to American option prices is computationally costly and technically challenging. In contrast, calibrating simple e.g. single-parameter models to American option prices is feasible. Yet fitting a singleparameter model to a whole option surface is not expected to yield useful results. One can, however, expect that a well-chosen single-parameter model can represent a single observed option price very well. This line of thoughts is exploited in the de-Americanization strategy by a multi-stage calibration procedure. First, for each pair of strike and maturity the corresponding observed American option price is used to fit a single-parameter model. Second, the European option prices in all of the resulting single-parameter models are computed. This gives a surface of pseudo-European option prices. This part of the procedure can be seen as a data pre-processing step where the observed prices are transferred into a more accessible data set, namely European option prices. The advantage is that in the second phase, the actual calibration is performed as a calibration to observed European option prices, for which a rich set of feasible and efficient tools is available. Putting this into more technical terms, given an input data of American put options, we consider the minimization problem (2.1) with $J(\boldsymbol{\mu}) \approx \widetilde{J}(\boldsymbol{\mu})$,

$$
\min _{\boldsymbol{\mu}} \widetilde{J}(\boldsymbol{\mu}), \quad \widetilde{J}(\boldsymbol{\mu}):=\frac{1}{M} \sum_{i=1}^{M}\left|\widetilde{P}_{i}^{\text {obs }}-P_{i}(\boldsymbol{\mu})\right|^{2},
$$

where the prices $\widetilde{P}_{j}^{\text {obs }}, j=1, \ldots, M$, are the pseudo-European put prices. These are obtained by perturbing the American put prices $P_{j}^{\text {obs }}$, i.e., $\widetilde{P}_{j}^{\text {obs }}:=D\left(P_{j}^{\text {obs }}\right)$, where $D$ : $\mathbb{R}^{M} \rightarrow \mathbb{R}^{M}$, and the corresponding prices $P_{i}(\boldsymbol{\mu})$ are the European put prices. As simple single-index model in the data-preprocessing phase, we use the binomial tree model of Cox et al. (1979) as in Burkovska et al. (2018). In other words, we use the binomial tree to transform each American option price into a pseudo-European option price. In a nutshell, once the observed market prices $P_{j}^{\text {obs }}, j=1, \ldots, M$, have been collected, for each single stock option an individual binomial tree is calibrated to match this option price $P_{j}^{\text {obs }}$. The resulting tree is used to price the associated so-called pseudo-European option with the same strike and maturity. A detailed description of the de-Americanization method is given in (Burkovska et al., 2018, Algorithm 1).

The advantage of this method is that the complexity of the non-linear problem for pricing American options can be reduced to that of the linear problem for pricing European options, allowing closed-form solutions or Fourier techniques to be exploited. The concept is highly attractive for the financial industry since the complex computational problem is reduced to standard ingredients that are typically available, well tested, heavily used and maintained in 
their computational library. The method is currently a market standard. We note that from the computational point of view, the DAS is very attractive, particularly in combination with the closed-form solutions. However, for each set of observations, an additional preprocessing time to transform the American data into European one is required, and, as we will see later, this step can dominate the computational cost of the entire calibration routine.

\section{Reduced Basis method (RBM)}

Reduced basis methods have become very popular in numerical analysis. Today they are still less common in finance. We therefore introduce the concept carefully. We start by presenting the PDE framework along with the variational formulation of the problem followed by the standard FEM discretization that we apply. Finally, we introduce the complexity reduction which consists in establishing a basis that is adapted to the parametric PDE problem.

4.1. PDE framework. By means of Itô calculus the option pricing problem can be recast as a PDE, see, e.g., Achdou and Pironneau (2005a). More precisely, let $P(\boldsymbol{\mu})$ denote a price of the European option, then $P(\boldsymbol{\mu})$ solves the following $\boldsymbol{\mu}$ PDEs:

$$
\begin{aligned}
\frac{\partial P(\boldsymbol{\mu})}{\partial \tau}+\mathcal{L}(\boldsymbol{\mu}) P(\boldsymbol{\mu}) & =0, & & \text { in }[0, T) \times \mathbb{R}_{+}^{n}, \\
P(T ; \boldsymbol{\mu}) & =\mathcal{H}, & & \text { in } \mathbb{R}_{+}^{n},
\end{aligned}
$$

where $\mathcal{H}$ is a pay-off functional $\left(\mathcal{H}(S)=(K-S)_{+}\right.$for the put and $\mathcal{H}(S)=(S-K)_{+}$for the call), $n=1,2$, and $\tau$ denotes the time to maturity $T$. The operator $\mathcal{L}$ is a second order linear differential operator of a convection-diffusion reaction type, and is defined by the model used to price the option, e.g., Black-Scholes, Black and Scholes (1973), CEV, Heston, Heston (1993). In particular, for the Heston model, the parameter $\boldsymbol{\mu}:=(\xi, \rho, \gamma, \kappa, r)$, and the operator $\mathcal{L}(\boldsymbol{\mu})$ is defined as follows

$$
\begin{aligned}
\mathcal{L}(\boldsymbol{\mu}) P(\boldsymbol{\mu}):=\frac{1}{2} \nu S^{2} \frac{\partial^{2} P(\boldsymbol{\mu})}{\partial S^{2}}+\xi \nu \rho S \frac{\partial^{2} P(\boldsymbol{\mu})}{\partial \nu \partial S}+\frac{1}{2} \xi^{2} \nu \frac{\partial^{2} P(\boldsymbol{\mu})}{\partial \nu^{2}} & +r S \frac{\partial P(\boldsymbol{\mu})}{\partial S} \\
& +\kappa(\gamma-\nu) \frac{\partial P(\boldsymbol{\mu})}{\partial \nu}-r P(\boldsymbol{\mu}) .
\end{aligned}
$$

In the case of American put options, an early exercise property of the option is incorporated by additional inequality constraints. Denoting by $P(\boldsymbol{\mu})$ the price of an American put option, we have that $P(\boldsymbol{\mu})$ satisfies

$$
\begin{aligned}
\frac{\partial P(\boldsymbol{\mu})}{\partial \tau}+\mathcal{L}(\boldsymbol{\mu}) P(\boldsymbol{\mu}) & \leq 0, & & \text { in }[0, T) \times \mathbb{R}_{+}^{n}, \\
P(\boldsymbol{\mu}) & \geq \mathcal{H}, & & \text { in } \quad[0, T) \times \mathbb{R}_{+}^{n}, \\
\left(\frac{\partial P(\boldsymbol{\mu})}{\partial \tau}+\mathcal{L}(\boldsymbol{\mu}) P(\boldsymbol{\mu})\right)(P(\boldsymbol{\mu})-\mathcal{H}) & =0, & & \text { in }[0, T) \times \mathbb{R}_{+}^{n}, \\
P(T ; \boldsymbol{\mu}) & =\mathcal{H}, & & \text { in } \quad \mathbb{R}_{+}^{n},
\end{aligned}
$$

with $\mathcal{H}(S):=(K-S)_{+}$.

Concerning the mathematical theory, we remark that, if complemented with suitable boundary conditions, the problems (4.1) and (4.2) typically admit unique solutions. However, while in the linear case (4.1) we can expect classical solutions, for the set of inequalities (4.2) one has to resort to a more general concept such as of weak solutions. We do 
not go into further details here, since, for the purposes of the paper, we consider a further approximation of the problem on a bounded domain.

4.2. Truncation to a bounded domain. In the present work, in order to build the approximation methods such as finite elements or the reduced basis method, we recast the PDE problem in a variational form, and work with the corresponding weak solutions. However, we do not work with (4.1)-(4.2) directly, but instead, we perform a further truncation of the PDE to a bounded domain $\Omega \subset \mathbb{R}^{2}$ with Lipschitz continuous boundary $\partial \Omega=\Gamma_{\mathrm{D}} \cup \Gamma_{\mathrm{N}}$, where $\Gamma_{\mathrm{D}}$ corresponds to the non-trivial Dirichlet part of the boundary $\partial \Omega$, i.e., $\Gamma_{D} \neq \emptyset$, and $\Gamma_{\mathrm{N}}$ stands for the part associated with the natural boundary conditions. Furthermore, we impose suitable Dirichlet and natural boundary conditions on these subdomains. Additionally, the operator $\mathcal{L}$ is typically degenerate for $S=0$, and it is standard practice to perform a $\log$-transformation of $S$ by introducing a new variable $x:=\log (S / K)$. Together with a log-transformation of $S$, we transform the PDE into a forward problem in time and we define $t:=T-\tau$. Then we denote by $w(t, x, \boldsymbol{\mu}):=P\left(T-t, K e^{x} ; \boldsymbol{\mu}\right)$ the option price in the new variables, and by $\chi(x):=\left(K-K e^{x}\right)+$ the pay-off functional for a put $\left(\right.$ or $\chi(x):=\left(K e^{x}-K\right)_{+}$for a call).

In the transformed variables, we can write the operator for the Heston model as

$$
\mathcal{L}(\boldsymbol{\mu}) w=-\nabla \cdot(\mathbf{A}(\boldsymbol{\mu}) \nabla w)+\mathbf{b}(\boldsymbol{\mu}) \nabla w+r w,
$$

where $\nabla:=\left(\frac{\partial}{\partial \nu}, \frac{\partial}{\partial x}\right)^{T}$, and the diffusion matrix and the velocity vector are given by

$$
\mathbf{A}(\boldsymbol{\mu}):=\frac{1}{2} \nu\left[\begin{array}{cc}
\xi^{2} & \rho \xi \\
\rho \xi & 1
\end{array}\right], \quad \mathbf{b}(\boldsymbol{\mu}):=\left[\begin{array}{c}
-\kappa(\gamma-\nu)+\frac{1}{2} \xi^{2} \\
-r+\frac{1}{2} \nu+\frac{1}{2} \xi \rho
\end{array}\right]
$$

The computational domain $\Omega$ for the Heston model is defined as follows:

$$
\Omega:=\left(\nu_{\min }, \nu_{\max }\right) \times\left(x_{\min }, x_{\max }\right) \subset \mathbb{R}^{2}, \quad \text { with } \quad x_{\min }<0<x_{\max }, \quad 0<\nu_{\min }<\nu_{\max } .
$$

Here, we follow the approach already used in, e.g., Kunoth et al. (2012); Winkler et al. (2001). In particular, $\nu=\nu_{\min }>0$, helps to avoid the degeneracy of the PDE at $\nu=0$. On $\partial \Omega$ we impose suitable boundary conditions. We note that for the Heston model several boundary conditions have been proposed in the literature. Here, we follow a functional analytic set up of the problem, which provides an existence and uniqueness of the weak solution. In particular, we consider Dirichlet together with the natural boundary conditions which arise from the weak formulation. In particular, for European put options, we consider the boundary conditions similar as in Düring and Fournié (2012),

$$
\begin{aligned}
& \frac{\partial w}{\partial n_{L}}(t, \nu, x)=0, \quad \text { on } \quad \Gamma_{\mathrm{N}}:=\left\{(\nu, x) \in \Omega: \nu \in\left\{\nu_{\min }, \nu_{\max }\right\}\right\}, \\
& w(t, \nu, x)=K e^{-r t}, \quad \text { on } \quad \Gamma_{\mathrm{D}}^{1}:=\left\{(\nu, x) \in \Omega: x=x_{\min }\right\}, \\
& w(t, \nu, x)=0, \quad \text { on } \quad \Gamma_{\mathrm{D}}^{2}:=\left\{(\nu, x) \in \Omega: x=x_{\max }\right\},
\end{aligned}
$$

where $\partial w / \partial n_{L}:=\mathbf{A}(\boldsymbol{\mu}) \nabla w \cdot \mathbf{n}$ is the conormal derivative of $w$, and $\mathbf{n}$ is the outward unit normal to $\partial \Omega$. For American put options, we specify the boundary conditions similar as proposed in Clarke and Parrott (1999); Düring and Fournié (2012),

$$
w(t, \nu, x)=\chi(x), \quad \text { on } \quad \Gamma_{\mathrm{D}}:=\Gamma_{\mathrm{D}}^{1} \cup \Gamma_{\mathrm{D}}^{2}, \quad \frac{\partial w}{\partial n_{L}}(t, \nu, x)=0, \quad \text { on } \quad \Gamma_{\mathrm{N}} .
$$

We note that we choose the truncated boundaries $x_{\min }, x_{\max }, \nu_{\min }, \nu_{\max }$ remote enough such that the truncation of the boundary does not influence the value of the option in the region of pricing interest. 
4.3. Variational formulation. Based on the truncated problem, we are ready to state the variational formulation of the problem which is the base for the development of the discrete approximation techniques.

We denote by $L^{2}(\Omega)$ a space of square integrable functions on $\Omega$, endowed with the corresponding inner product and norm, denoted by $(\cdot, \cdot)_{L^{2}(\Omega)}$ and $\|\cdot\|_{L^{2}(\Omega)}$, respectively. We denote by $H^{1}(\Omega)$ a usual Sobolev space and introduce the following functional spaces

$$
X=H^{1}(\Omega):=\left\{v \in L^{2}(\Omega): \nabla v \in L^{2}(\Omega)\right\}, \quad V:=\left\{v \in X:\left.v\right|_{\Gamma_{\mathrm{D}}}=0\right\},
$$

equipped with the norms $\|v\|_{X}^{2}=\|v\|_{H^{1}(\Omega)}^{2}:=\|\nabla v\|_{L^{2}(\Omega)}+\|v\|_{L^{2}(\Omega)}$ and $\|v\|_{V}^{2}=|v|_{H^{1}(\Omega)}^{2}:=$ $\|\nabla v\|_{L^{2}(\Omega)}$, which correspond to the $H^{1}(\Omega)$ norm and semi-norm respectively. Let $V^{\prime}$ be the dual space of $V$, and denote by $\langle\cdot, \cdot\rangle$ the duality pairing of $V$ with $V^{\prime}$. We then introduce a bilinear form, $a: V \times V \times \mathcal{P} \rightarrow \mathbb{R}$, which is derived from the differential operator $\mathcal{L}$, and for the case of the Heston model is defined as

$$
a(u, v ; \boldsymbol{\mu}):=\int_{\Omega} \mathbf{A}(\boldsymbol{\mu}) \nabla u \cdot \nabla v+\int_{\Omega} \mathbf{b}(\boldsymbol{\mu}) \cdot \nabla u v+\int_{\Omega} r u v,
$$

where $\mathbf{A}(\boldsymbol{\mu})$ and $\mathbf{b}(\boldsymbol{\mu})$ are specified in (4.3). Note that $\nu \geq \nu_{\min }>0, \rho \in(-1,1)$ and hence $\mathbf{A}(\boldsymbol{\mu})$ is positive definite on $\bar{\Omega}$. It follows from the admissible values of the parameters that for all $\boldsymbol{\mu} \in \mathcal{P}$ the bilinear form $a(\cdot, \cdot ; \boldsymbol{\mu})$ is continuous and satisfies a Gårding inequality on $V \times V$. That is, there exist constants $0<\bar{\alpha}_{a} \leq \alpha_{a}(\boldsymbol{\mu}), 0<\gamma_{a}(\boldsymbol{\mu}) \leq \bar{\gamma}_{a}<\infty$, $0 \leq \lambda_{a}(\boldsymbol{\mu}) \leq \bar{\lambda}_{a}<\infty$, such that

$$
\begin{aligned}
|a(u, v ; \boldsymbol{\mu})| & \leq \gamma_{a}(\boldsymbol{\mu})\|u\|_{V}\|v\|_{V} \quad \forall u, v \in V, & & \text { (continuity) } \\
a(v, v ; \boldsymbol{\mu}) & \geq \alpha_{a}(\boldsymbol{\mu})\|v\|_{V}^{2}-\lambda_{a}(\boldsymbol{\mu})\|v\|_{L^{2}(\Omega)}^{2} & \forall v \in V . & \text { (Gårding inequality) }
\end{aligned}
$$

To tackle non-homogeneous Dirichlet boundary conditions, we denote by $u_{L}(\boldsymbol{\mu})$ a continuous extension of the Dirichlet data to the interior of the domain. We can express $w(t ; \boldsymbol{\mu})=u(t ; \boldsymbol{\mu})+u_{L}(t ; \boldsymbol{\mu})$, where $u$ solves the problem with homogeneous Dirichlet boundary conditions with $u(0 ; \boldsymbol{\mu})=u_{0}(\boldsymbol{\mu})=w(0 ; \boldsymbol{\mu})-u_{L}(0 ; \boldsymbol{\mu})$.

Then the weak form corresponding to the European option pricing problem reads: Given $u_{0}(\boldsymbol{\mu}) \in L^{2}(\Omega), f(\boldsymbol{\mu}) \in L^{2}\left(0, T ; V^{\prime}\right), \boldsymbol{\mu} \in \mathcal{P}$, find $u(\boldsymbol{\mu}) \in L^{2}(0, T ; V) \cap H^{1}\left(0, T ; V^{\prime}\right)$ such that for a.e. $t \in(0, T)$ and all $\boldsymbol{\mu} \in \mathcal{P}$ holds

$$
\begin{aligned}
\left\langle\frac{\partial u}{\partial t}(t ; \boldsymbol{\mu}), v\right\rangle+a(u(t ; \boldsymbol{\mu}), v ; \boldsymbol{\mu}) & =\langle f(t ; \boldsymbol{\mu}), v\rangle, \quad \forall v \in V, \\
u(0) & =u_{0}(\boldsymbol{\mu}),
\end{aligned}
$$

where $\langle f(t ; \boldsymbol{\mu}), v\rangle=-\left\langle\frac{\partial u_{L}}{\partial t}(t ; \boldsymbol{\mu}), v\right\rangle-a\left(u_{L}(t ; \boldsymbol{\mu}), v ; \boldsymbol{\mu}\right)$. The above problem is well-posed, see, e.g., (Quarteroni and Valli, 1994, Theorem 11.1.1, Remark 11.1.1). To recast the American put price PDE in a variational form we introduce the following set of admissible solutions

$$
\mathcal{K}(t ; \boldsymbol{\mu}):=\{v \in V, \quad v \geq \widetilde{\chi}(t ; \boldsymbol{\mu}) \text { in } \Omega\}, \quad \text { a.e. } t \in[0, T]
$$

where $\tilde{\chi}(t ; \boldsymbol{\mu})=\chi-u_{L}(t ; \boldsymbol{\mu})$. Then the weak formulation of the American put price PDE leads to the following variational inequality problem: Given $u_{0}(\boldsymbol{\mu}) \in \mathcal{K}(\boldsymbol{\mu}), f(\boldsymbol{\mu}), f^{\prime}(\boldsymbol{\mu}) \in$ $L^{2}\left(0, ; V^{\prime}\right), \boldsymbol{\mu} \in \mathcal{P}$, find $u(\boldsymbol{\mu}) \in L^{2}(0, T ; V) \cap H^{1}\left(0, T ; V^{\prime}\right)$, such that $u(t ; \boldsymbol{\mu}) \in \mathcal{K}(t ; \boldsymbol{\mu})$ for 
a.e. $t \in(0, T)$ and for all $\boldsymbol{\mu} \in \mathcal{P}$ satisfies

$$
\begin{aligned}
\left\langle\frac{\partial u}{\partial t}(t ; \boldsymbol{\mu}), v\right\rangle+a(u(t ; \boldsymbol{\mu}), v-u(t ; \boldsymbol{\mu}) ; \boldsymbol{\mu}) & \geq\langle f(t ; \boldsymbol{\mu}), v-u(t ; \boldsymbol{\mu})\rangle, \quad \forall v \in \mathcal{K}(t ; \boldsymbol{\mu}), \\
u(0) & =u_{0}(\boldsymbol{\mu}) .
\end{aligned}
$$

The variational inequality problem (4.10) admits a unique solution, see, e.g., (Glowinski et al., 1981, Chapter 6, Theorem 2.1).

4.4. Discretization of the PDE by finite-differences in time and finite elements in space. For the temporal discretization the $\theta$-scheme is used, $\theta \in[0,1]$ with $\theta=1$, $\theta=1 / 2$ corresponding to the implicit Euler and Crank-Nicolson methods respectively. We subdivide the time interval $[0, T]$ into $I$ subintervals of equal length, $t^{k}:=k \Delta t, 0<k \leq I$ with $\Delta t=T / I$. For all $\boldsymbol{\mu} \in \mathcal{P}, 0 \leq k \leq I$, we introduce the notation $w^{k}(\boldsymbol{\mu})=u^{k}(\boldsymbol{\mu})+$ $u_{L}^{k}(\boldsymbol{\mu}):=w\left(t^{k}, \boldsymbol{\mu}\right), u^{k}(\boldsymbol{\mu}) \in V, u_{L}^{k}(\boldsymbol{\mu}) \in X$, and $\widetilde{\chi}^{k}(\boldsymbol{\mu}):=\widetilde{\chi}\left(t^{k} ; \boldsymbol{\mu}\right)=\chi-u_{L}^{k}(\boldsymbol{\mu})$. Then for a given $\boldsymbol{\mu} \in \mathcal{P}, \theta \in[0,1], u^{0}(\boldsymbol{\mu})=u_{0}(\boldsymbol{\mu})$, we arrive in the following semi-discrete version of (4.9): Find $u^{k+1}(\boldsymbol{\mu}) \in V, 0 \leq k \leq I-1$, such that

$$
\frac{1}{\Delta t}\left(u^{k+1}-u^{k}, v\right)+a\left(\theta u^{k+1}+(1-\theta) u^{k}, v ; \boldsymbol{\mu}\right)=f^{k}(v ; \boldsymbol{\mu}), \quad v \in V,
$$

where $f^{k}(v ; \boldsymbol{\mu}):=-\frac{1}{\Delta t}\left(u_{L}^{k+1}-u_{L}^{k}, v\right)+a\left(\theta u_{L}^{k+1}+(1-\theta) u_{L}^{k}, v ; \boldsymbol{\mu}\right)$.

By means of a Lagrange multiplier, the variational inequality problem (4.10), we reformulate in an equivalent mixed problem or a so-called saddle point problem, see, e.g., (Kikuchi and Oden, 1988, Chapter 3). The advantage of this formulation is that instead of constructing a conforming approximation of the convex set $\mathcal{K}$ we will deal with an approximation of the linear space. Define a bilinear form $b: V^{\prime} \times V \rightarrow \mathbb{R}$ as a duality pairing $b(\eta, v):=\langle\eta, v\rangle$, $\eta \in V^{\prime}, v \in V$. We introduce a set $M \subset V^{\prime}$ (referred as a dual cone), defined as

$$
M:=\left\{\eta \in V^{\prime}: \quad b(\eta, v) \geq 0, v \in V, v \geq 0\right\} .
$$

Then we consider the following semi-discrete weak saddle point problem: For $\boldsymbol{\mu} \in \mathcal{P}$, $0 \leq k \leq I-1, \theta \in[0,1]$, find $u^{k+1}(\boldsymbol{\mu}) \in V, \lambda^{k+1}(\boldsymbol{\mu}) \in M$, satisfying

$$
\begin{array}{rlrl}
\frac{1}{\Delta t}\left(u^{k+1}-u^{k}, v\right)+a\left(\theta u^{k+1}+(1-\theta) u^{k}, v ; \boldsymbol{\mu}\right)-b\left(\lambda^{k+1}, v\right) & =f^{k}(v ; \boldsymbol{\mu}), & & v \in V, \\
b\left(\eta-\lambda^{k+1}, u^{k+1}-\tilde{\chi}^{k+1}\right) \geq 0, & & \eta \in M .
\end{array}
$$

Note that for all $\boldsymbol{\mu} \in \mathcal{P}, 0 \leq k \leq I-1, f^{k} \in V^{\prime}$ and the bilinear form $a(\cdot, \cdot ; \boldsymbol{\mu})$ is continuous and satisfies the Gårding inequality. Thus, for a small enough time step $\Delta t<\left(1 / \theta \lambda_{a}(\boldsymbol{\mu})\right)$, by a generalized Lax-Milgram argument, the problem (4.11) admits a unique solution $u(\boldsymbol{\mu}) \in V$, see, e.g., (Quarteroni and Valli, 1994, Theorem 5.1.1). For the variational inequality problem (4.13) to be well-posed we additionally need to guarantee that $b(\cdot, \cdot)$ satisfies the inf-sup stability condition on $V^{\prime} \times V$, i.e., there exists $\beta_{0}>0$ such that $\inf _{\eta \in V^{\prime}} \sup _{v \in V} b(\eta, v) /\|\eta\|_{V^{\prime}}\|v\|_{V} \geq \beta_{0}>0$. For the present choices of the functional spaces we have $\beta_{0}=1$, and unique solvability of (4.13) is given, see, e.g., (Brezzi et al., 1978, Theorem 2.1).

Next we consider a spatial discretization of the presented problems. We approximate the solution spaces $X, V, V^{\prime}$ by finite dimensional subspaces $X_{\mathcal{N}} \subset X, V_{\mathcal{N}} \subset V, V_{\mathcal{N}}^{\prime} \subset V^{\prime}$ of the dimensions $\mathcal{N}_{X}, \mathcal{N}_{V}$ and $\mathcal{N}_{V}^{\prime}$ respectively:

$$
X_{\mathcal{N}}=\operatorname{span}\left\{\phi_{p}\right\}_{p=1}^{\mathcal{N}_{X}}, \quad V_{\mathcal{N}}=X_{\mathcal{N}} \cap V=\operatorname{span}\left\{\phi_{p}\right\}_{p=1}^{\mathcal{N}_{V}}, \quad V_{\mathcal{N}}^{\prime}=\operatorname{span}\left\{\zeta_{p}\right\}_{p=1}^{\mathcal{N}_{V}^{\prime}} .
$$


The discrete Lagrange multiplier cone $M_{\mathcal{N}} \subset V_{\mathcal{N}}^{\prime}$ is defined as

$$
M_{\mathcal{N}}=\operatorname{span}_{+}\left\{\zeta_{q}\right\}_{q=1}^{\mathcal{N}_{V}^{\prime}}:=\left\{\eta \in V_{\mathcal{N}}: \quad \eta=\sum_{q=1}^{\mathcal{N}_{V}^{\prime}} \alpha_{q} \zeta_{q}, \quad \alpha_{q} \geq 0\right\}
$$

For a given $\boldsymbol{\mu} \in \mathcal{P}$ and $0 \leq k \leq I-1$, the solution pair $\left(u^{k+1}(\boldsymbol{\mu}), \lambda^{k+1}(\boldsymbol{\mu})\right) \in V \times M$ is then approximated by $\left(u_{\mathcal{N}}^{k+1}(\boldsymbol{\mu}), \lambda_{\mathcal{N}}^{k+1}(\boldsymbol{\mu})\right) \in V_{\mathcal{N}} \times M_{\mathcal{N}}$, where

$$
u_{\mathcal{N}}^{k+1}(\boldsymbol{\mu})=\sum_{p=1}^{\mathcal{N}_{V}} u_{\mathcal{N}, p}^{k+1}(\boldsymbol{\mu}) \phi_{p}, \quad \lambda_{\mathcal{N}}^{k+1}(\boldsymbol{\mu})=\sum_{q=1}^{\mathcal{N}_{V}^{\prime}} \lambda_{\mathcal{N}, q}^{k+1}(\boldsymbol{\mu}) \zeta_{q}
$$

The Dirichlet lift function $u_{L}^{k}(\boldsymbol{\mu}) \in X$ is approximated by the discrete lift function $u_{L \mathcal{N}}^{k}(\boldsymbol{\mu}) \in$ $X_{\mathcal{N}}$, which can be chosen as, e.g., a nodal interpolation of the Dirichlet datum. Accordingly, a discrete modified pay-off $\widetilde{\chi}_{\mathcal{N}}^{k}(\boldsymbol{\mu}) \in X_{\mathcal{N}}$ approximates $\widetilde{\chi}^{k}(\boldsymbol{\mu})$ and we set $u_{\mathcal{N}}^{0}(\boldsymbol{\mu})=\widetilde{\chi}_{\mathcal{N}}^{0}(\boldsymbol{\mu})$. Then, for the European put options $u_{\mathcal{N}}^{k+1}(\boldsymbol{\mu}) \in V_{\mathcal{N}}, \boldsymbol{\mu} \in \mathcal{P}, 0 \leq k \leq I-1$, solves the following discrete problem

$$
\mathbb{E}_{\mathcal{N}}^{\mathrm{Eu}}(\boldsymbol{\mu})=\left\{\begin{array}{l}
\frac{1}{\Delta t}\left(u_{\mathcal{N}}^{k+1}-u_{\mathcal{N}}^{k}, v\right)+a\left(\theta u_{\mathcal{N}}^{k+1}+(1-\theta) u_{\mathcal{N}}^{k}, v ; \boldsymbol{\mu}\right)=f^{k}(v ; \boldsymbol{\mu}), \\
\left(u_{\mathcal{N}}^{0}-u^{0}, v\right)_{V}=0, \quad v \in V_{\mathcal{N}}
\end{array}\right.
$$

The solution pair $\left(u_{\mathcal{N}}^{k+1}(\boldsymbol{\mu}), \lambda_{\mathcal{N}}^{k+1}(\boldsymbol{\mu})\right) \in V_{\mathcal{N}} \times M_{\mathcal{N}}, \boldsymbol{\mu} \in \mathcal{P}, 0 \leq k \leq I-1$, for the American put option problem in turn satisfies the following saddle point problem

$$
\mathbb{E}_{\mathcal{N}}^{\mathrm{Am}}(\boldsymbol{\mu})=\left\{\begin{array}{l}
\frac{1}{\Delta t}\left(u_{\mathcal{N}}^{k+1}-u_{\mathcal{N}}^{k}, v\right)+a\left(\theta u_{\mathcal{N}}^{k+1}+(1-\theta) u_{\mathcal{N}}^{k}, v ; \boldsymbol{\mu}\right)-b\left(\lambda_{\mathcal{N}}^{k+1}, v\right)=f^{k}(v ; \boldsymbol{\mu}) \\
b\left(\eta-\lambda_{\mathcal{N}}^{k+1}, u_{\mathcal{N}}^{k+1}-\tilde{\chi}_{\mathcal{N}}^{k+1}\right) \geq 0 \\
\left(u_{\mathcal{N}}^{0}-u^{0}, v\right)_{V}=0
\end{array}\right.
$$

Due to the conformity of the discrete spaces the continuity and coercivity (or Gårding inequality) of the bilinear forms are carried over to the discrete spaces, that ensures the well-posedness of the discrete problem $\mathbb{E}_{\mathcal{N}}^{\mathrm{Eu}}(\boldsymbol{\mu})$. In contrast, for the saddle-point problem $\mathbb{E}_{\mathcal{N}}^{\mathrm{Am}}(\boldsymbol{\mu})$, the inf-sup stability of $b(\cdot, \cdot)$ does not follow directly. In our numerical experiments in Section 6 we use discontinuous piecewise linear biorthogonal basis functions Wohlmuth (2000) to discretize the dual space $V^{\prime}$, and standard piecewise linear continuous finite element basis functions to approximate the primal space $V$. This setting of discrete spaces ensures the inf-sup stability condition, see Wohlmuth (2000).

4.5. Complexity reduction by the reduced basis method (RBM). The high-fidelity discrete problems $\mathbb{E}_{\mathcal{N}}^{\mathrm{Eu} / \mathrm{Am}}(\boldsymbol{\mu})$ (which are often called in the reduced basis literature "the detailed problems"), in general, are computationally expensive for large $\mathcal{N}_{V}$, and significantly slow down the calibration procedure. To reduce the complexity, we apply the reduced basis method. The basis idea of the RBM is to construct the reduced-order surrogate model $\mathbb{E}_{N}^{\mathrm{Eu} / \mathrm{Am}}(\boldsymbol{\mu})$, which can approximate the original high-fidelity problem $\mathbb{E}_{\mathcal{N}}^{\mathrm{Eu} / \mathrm{Am}}(\boldsymbol{\mu})$ for different values of $\boldsymbol{\mu} \in \mathcal{P}$ at a low computational cost. The reduced model is constructed based on an approximation of $V_{\mathcal{N}}, V_{\mathcal{N}}^{\prime}, M_{\mathcal{N}}$ by low-dimensional reduced spaces $V_{N} \subset V_{\mathcal{N}}$ for European options and $V_{N} \subset V_{\mathcal{N}}, V_{N}^{\prime} \subset V_{\mathcal{N}}^{\prime}, M_{N} \subset M_{\mathcal{N}}$ for American options with $\operatorname{dim}\left(V_{N}\right) \ll \operatorname{dim}\left(V_{\mathcal{N}}\right), \operatorname{dim}\left(V_{N}^{\prime}\right) \ll \operatorname{dim}\left(V_{\mathcal{N}}^{\prime}\right)$. Then for a given $\boldsymbol{\mu} \in \mathcal{P}$, we approximate 
$u_{\mathcal{N}}^{k+1} \in V_{\mathcal{N}}$ by $u_{N}^{k+1}(\boldsymbol{\mu}) \in V_{N}, \lambda_{\mathcal{N}}^{k+1}(\boldsymbol{\mu}) \in M_{\mathcal{N}}$ by $\lambda_{N}^{k+1}(\boldsymbol{\mu}) \in M_{N}, 0 \leq k \leq I-1$. The reduced surrogate models for pricing European and American options are defined as follows

$$
\begin{aligned}
\mathbb{E}_{N}^{\mathrm{Eu}}(\boldsymbol{\mu})=\left\{\begin{array}{l}
\frac{1}{\Delta t}\left(u_{N}^{k+1}-u_{N}^{k}, v\right)_{L^{2}(\Omega)}+a\left(\theta u_{N}^{k+1}+(1-\theta) u_{N}^{k}, v ; \boldsymbol{\mu}\right)=f^{k}(v ; \boldsymbol{\mu}), \\
\left(u_{N}^{0}-u_{\mathcal{N}}^{0}, v\right)_{V}=0, \quad v \in V_{N} .
\end{array}\right. \\
\mathbb{E}_{N}^{\mathrm{Am}}(\boldsymbol{\mu})=\left\{\begin{array}{l}
\frac{1}{\Delta t}\left(u_{N}^{k+1}-u_{N}^{k}, v\right)_{L^{2}(\Omega)}+a\left(\theta u_{N}^{k+1}+(1-\theta) u_{N}^{k}, v ; \boldsymbol{\mu}\right)-b\left(\lambda_{N}^{k+1}, v\right)=f^{k}(v ; \boldsymbol{\mu}), \\
b\left(\eta-\lambda_{N}^{k+1}, u_{N}^{k+1}-\widetilde{\chi}_{\mathcal{N}}^{k+1}\right) \geq 0, \\
\left(u_{N}^{0}-u_{\mathcal{N}}^{0}, v\right)_{V}=0 .
\end{array}\right.
\end{aligned}
$$

Additionally, we require that the reduced spaces $V_{N}, V_{N}^{\prime}$ are constructed such that the bilinear form $b(\cdot, \cdot)$ is uniformly inf-sup stable on $V_{N}^{\prime} \times V_{N}$ with respect to $N$. Thus the well-posedness of the reduced problems $E_{N}^{\mathrm{Eu}}(\boldsymbol{\mu})$ and $E_{N}^{\mathrm{Am}}(\boldsymbol{\mu})$ is given, see also Burkovska et al. (2015); Haasdonk et al. (2012). The algebraic system of equations resulting from the discrete inequality problem can be solved by, e.g., a primal-dual-active set method. For more details on implementation and assembly of the reduced saddle point problem $\mathbb{E}_{N}^{\mathrm{Am}}(\boldsymbol{\mu})$ we refer to, e.g., Burkovska et al. (2015); Burkovska (2016).

Numerous approaches exist for the construction of the reduced basis approximation spaces. Their common goal is to exploit the parameter dependence of the problem and to incorporate this information into the construction of the reduced bases. Typically, this is done by applying an iterative greedy strategy to a set of snapshots, i.e., solutions computed for different parameter values. For linear parabolic problems, a popular choice is a combination of the greedy strategy for parameter selection and a proper orthogonal decomposition (POD) in time resulting in a so-called POD-Greedy algorithm Haasdonk (2013); Haasdonk and Ohlberger (2008). For parabolic variational inequalities, the construction is more challenging due to the requirement of uniform inf-sup stability. For stationary variational inequalities, a greedy sampling is commonly used, Haasdonk et al. (2012); Zhang et al. (2016), while for time-dependent problems a POD-Angle-Greedy Burkovska et al. (2015) and a POD-NNMF ${ }^{1}$ Balajewicz et al. (2016) have been considered in the literature. In the present work, we follow the idea of the POD-Greedy algorithm for European options and POD-Angle-Greedy algorithm for American options, presented in Algorithm 1, Section 4.6.

A computational speed-up of the reduced basis method is achieved by a so-called offline/online procedure, which relies on the assumption that the problem has an affine dependence on its parameters. That is, for every $\boldsymbol{\mu} \in \mathcal{P}$ the bilinear and linear forms are parameter separable, i.e., there exist $\Theta_{q}^{a}: \mathcal{P} \rightarrow \mathbb{R}, q=1, \ldots, Q_{a}$, such that $a(\cdot, \cdot ; \boldsymbol{\mu}):=$ $\sum_{q=1}^{Q_{a}} \Theta_{q}^{a}(\boldsymbol{\mu}) a_{q}(\cdot, \cdot)$, where $a_{q}: V \times V \rightarrow \mathbb{R}$ are parameter-independent. The same arguments apply to $f^{k}(\cdot ; \boldsymbol{\mu}), \widetilde{\chi}_{\mathcal{N}}^{k}(\boldsymbol{\mu})$ and $u_{\mathcal{N}}^{0}(\boldsymbol{\mu})$. For example, while $f(x ; \boldsymbol{\mu}):=\boldsymbol{\mu} \sin (\pi x)$ is affine in the parameter $\boldsymbol{\mu}$, the function $f(x ; \boldsymbol{\mu}):=\sin (\boldsymbol{\mu} \pi x)$ is not. We note that for the Heston model the bilinear from $a(\cdot, \cdot ; \boldsymbol{\mu})$ in (4.7) is affine in the parameter $\boldsymbol{\mu}$. For nonaffine in parameter or nonlinear problems, one can employ, e.g., an empirical interpolation method, see, e.g., Barrault et al. (2004), to resolve to the affine in parameter structure and preserve the efficiency of the RBM. Then an offline routine requires the evaluation of all parameter-independent quantities, e.g., $a_{q}(\cdot, \cdot), q=1, \ldots Q_{a}$. Usually this procedure is computationally cost-intense and depends on the dimension of the detailed problem $\mathcal{N}_{V}$, however it is performed only once. In turn, the online procedure is computationally cheap

\footnotetext{
${ }^{1}$ NNMF refers to a non-negative matrix factorization procedure, Lee and Seung (1999).
} 
and involves assembling the parameter-dependent components, e.g., $\Theta_{q}^{a}(\boldsymbol{\mu}), q=1, \ldots, Q_{a}$, and solving the reduced system. This stage is executed multiple times for each new parameter value $\boldsymbol{\mu} \in \mathcal{P}$; see, e.g., Hesthaven et al. (2016); Quarteroni et al. (2016) for more details. In Figure 1 we schematically demonstrate the computational procedure for the $\mathrm{RBM}$ in comparison to the standard FEM.

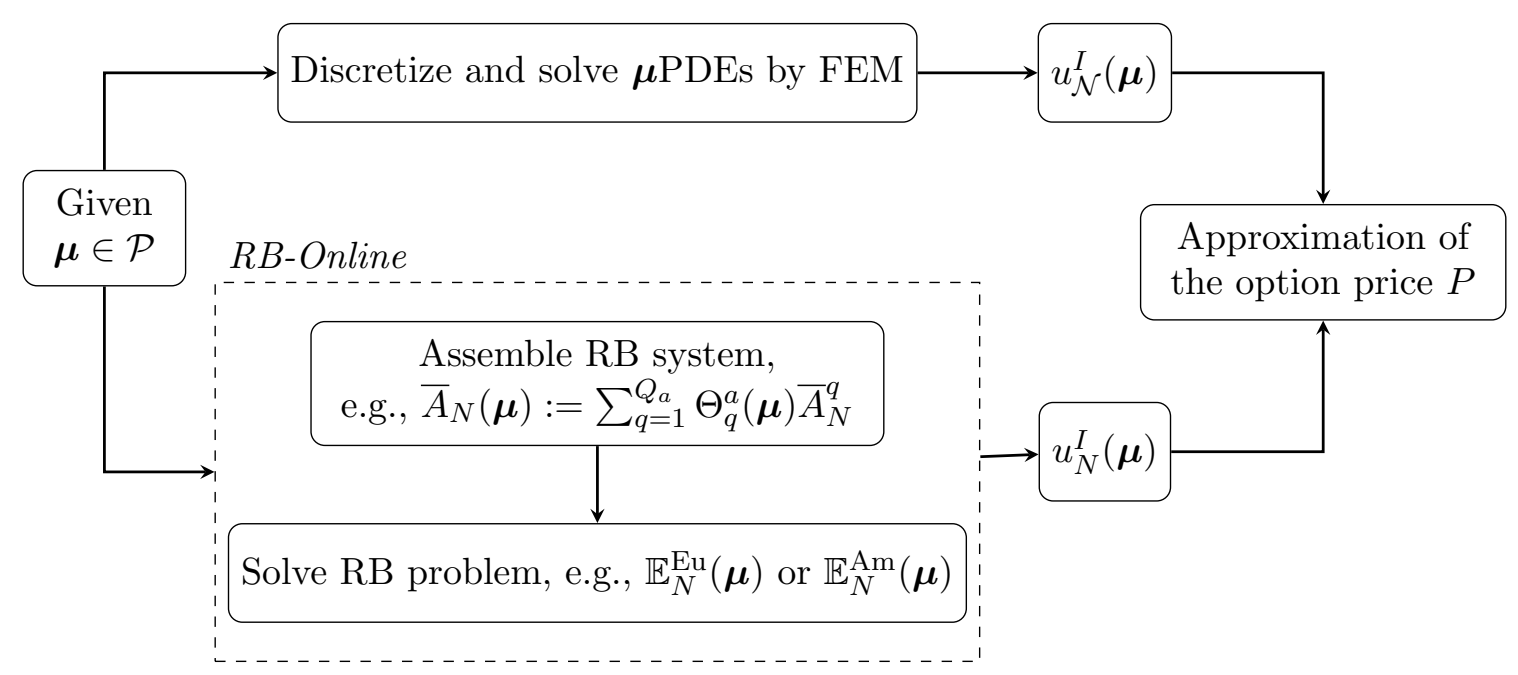

RB-Offline (evaluated once)

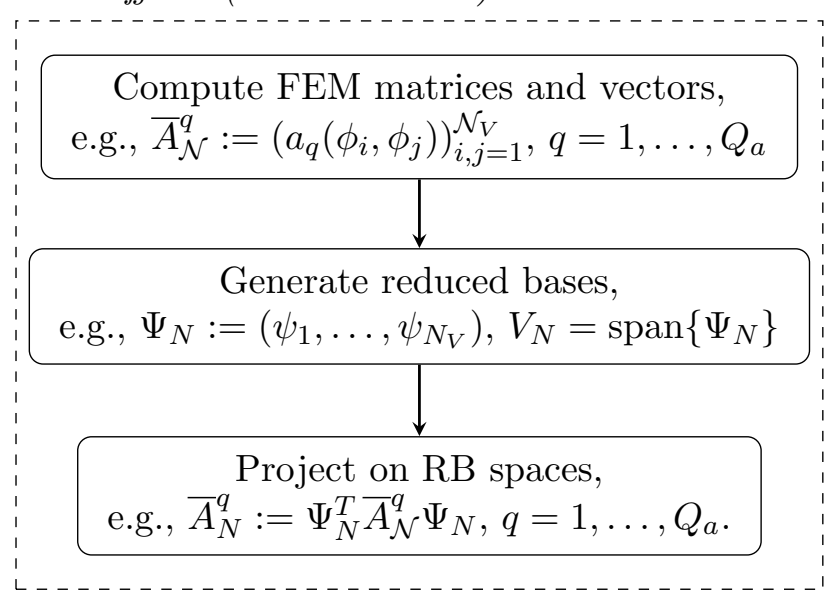

FIgURE 1. The FEM and RBM procedure for option pricing at a glance.

4.6. Reduced basis construction. Here we describe the algorithm used to construct the reduced basis spaces. Since the European option problem can be considered as a particular case of the American option problem formulation, we focus on the description of the basis construction for the latter problem and comment only on the differences.

Consider a finite subset $\mathcal{P}_{N}:=\left\{\boldsymbol{\mu}_{1}, \ldots, \boldsymbol{\mu}_{N}\right\}, \boldsymbol{\mu}_{i} \neq \boldsymbol{\mu}_{j}, \forall i \neq j, N \in \mathbb{N}$ and define the reduced spaces $V_{N}:=\operatorname{span}\left\{\Psi_{N}\right\}$ and $V_{N}^{\prime}:=\operatorname{span}\left\{\Xi_{N}\right\}$, where the primal $\Psi_{N}:=$ $\left\{\psi_{1}, \ldots, \psi_{N_{V}}\right\} \subset V_{\mathcal{N}}$, and the dual $\Xi_{N}:=\left\{\xi_{1}, \ldots, \xi_{N_{V^{\prime}}}\right\} \subset V_{\mathcal{N}}^{\prime}$ reduced bases are constructed from the large set of snapshot solutions $u_{\mathcal{N}}^{k}\left(\boldsymbol{\mu}_{i}\right)$ and $\lambda_{\mathcal{N}}^{k}\left(\boldsymbol{\mu}_{i}\right), 1 \leq k \leq I, i=$ 
$1, \ldots, N$. The reduced cone is defined as $M_{N}:=\operatorname{span}_{+}\left\{\xi_{j}\right\}_{j=1}^{N_{V^{\prime}}}:=\left\{\sum_{j=1}^{N_{V^{\prime}}} \alpha_{j} \xi_{j}, \alpha_{j} \geq 0\right\}$. By construction $\xi_{j} \in M_{\mathcal{N}}$ and thus $M_{N} \subset M_{\mathcal{N}}$.

The approach we follow to construct $\Psi_{N}$ and $\Xi_{N}$ is presented in Algorithm 1. We investigate the parameter domain $\mathcal{P}$, that is replaced by a finite set $\mathcal{P}_{\text {train }} \subset \mathcal{P}$, by a greedy search (Step 5-Step 13). In the greedy loop, we identify a "worst" parameter $\boldsymbol{\mu}_{N}$, i.e., a parameter which leads to the worst RB approximation, and add it to the training set. In other words, at each iteration of the algorithm we select $\boldsymbol{\mu}$ which maximizes $E_{N}(\boldsymbol{\mu})$, where $E_{N}(\boldsymbol{\mu})$ can be chosen, e.g., as a "true" error between the detailed and reduced solutions:

$$
E_{N}(\boldsymbol{\mu}):=\left(\sum_{k=0}^{I}\left\|u_{\mathcal{N}}^{k}(\boldsymbol{\mu})-u_{N}^{k}(\boldsymbol{\mu})\right\|_{V}^{2}\right)^{1 / 2} .
$$

Such selection procedure ensures that the error in this particular parameter will be reduced in the subsequent iterations of the algorithm.

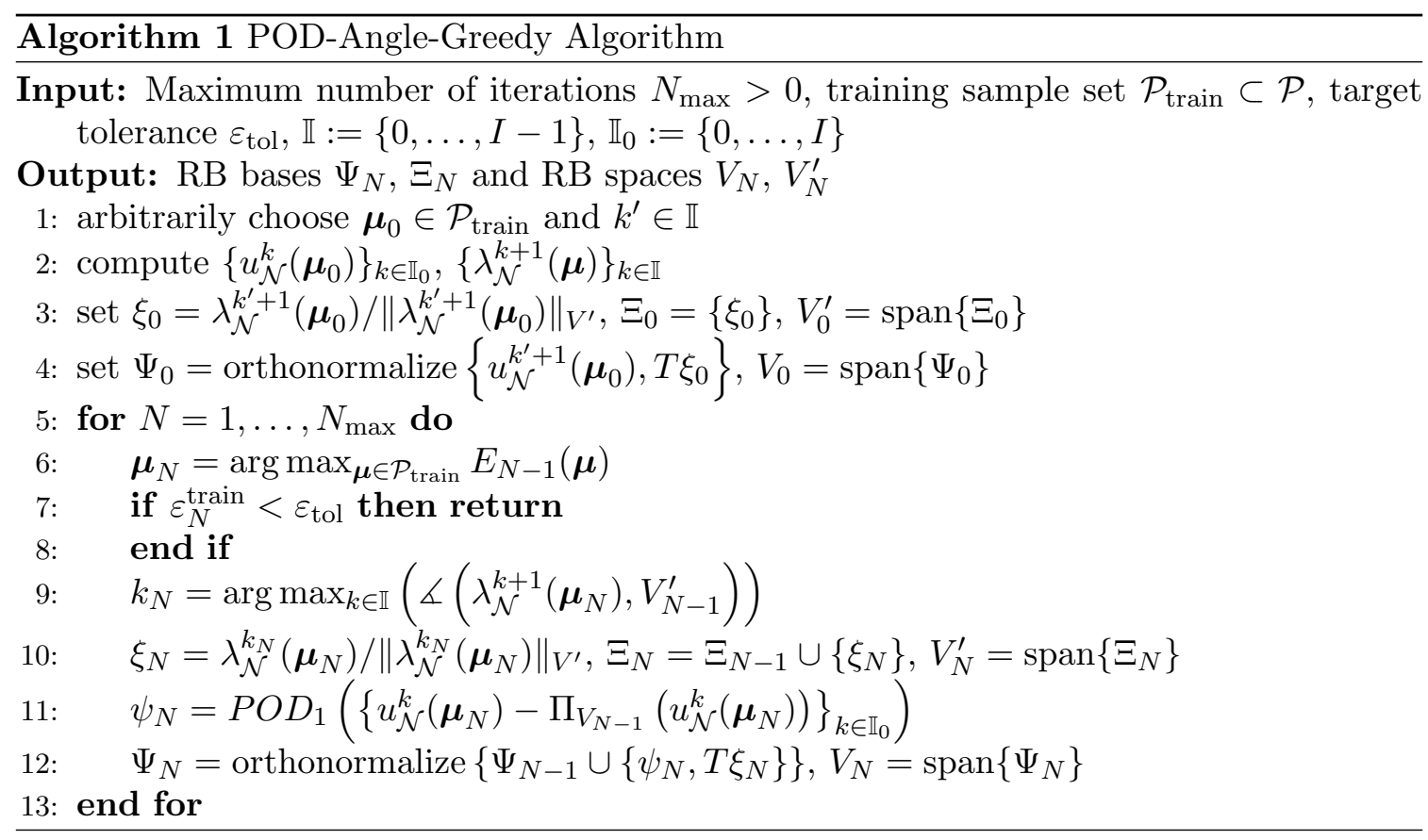

The error measure $E_{N}\left(\boldsymbol{\mu}_{N}\right)$ can be also set as an a posteriori error bound Burkovska et al. (2015); Haasdonk and Ohlberger (2008). The availability and efficient computation of the latter choice makes it more attractive from a computational point of view. Next, for the selected parameter, we compute primal and dual bases and repeat this process $N_{\max }$ times or until the desired tolerance $\varepsilon_{\text {tol }}$ of the stopping criterion is reached. For the primal reduced space construction, we apply the standard POD-Greedy procedure (Step 11), where the difference between the worst resolved trajectory $u_{\mathcal{N}}^{k}\left(\boldsymbol{\mu}_{N}\right), 0 \leq k \leq \mathbb{I}-1$, and its orthogonal projection onto the current RB space $\Pi_{V_{N-1}} u_{\mathcal{N}}^{k}\left(\boldsymbol{\mu}_{N}\right)$ is compressed to the first dominant POD mode:

$$
P O D_{1}\left(\left\{v^{k}\right\}_{k \in \mathbb{I}_{0}}\right):=\underset{\|z\|_{V}=1}{\arg \min } \sum_{k \in \mathbb{I}_{0}}\left\|v^{k}-\left(v^{k}, z\right)_{V} z\right\|_{V}^{2} .
$$

To construct the dual RB space, the vectors that maximize the volume of the resulting cone, i.e., vectors showing the largest deviation from the current RB space (Step 9, are 
selected. We denote $\measuredangle(\eta, Y):=\arccos \left(\left\|\Pi_{Y} \eta\right\|_{V^{\prime}} /\|\eta\|_{V^{\prime}}\right)$ the angle between the vector $\eta \in V^{\prime}$ and the linear space $Y \subset V^{\prime}$, where $\Pi_{Y} \eta$ is an orthogonal projection of $\eta$ onto $Y$.

Additionally, to form a uniformly stable pair of the reduced spaces $V_{N}, V_{N}^{\prime}$, we enrich the primal space $V_{N}$ by the "supremizers" $T \xi_{N}$, Rovas (2003); Rozza and Veroy (2007), where $T: V_{\mathcal{N}}^{\prime} \rightarrow V_{\mathcal{N}}$ is a "supremizing" operator, defined as a solution of $\left(T \xi_{N}, v\right)_{V}:=b\left(\xi_{N}, v\right)$, for all $v \in V_{\mathcal{N}}$. It is easy to see that this inclusion ensures the inf-sup stability of $b(\cdot, \cdot)$ on $V_{N}^{\prime} \times V_{N}$, see, e.g., Haasdonk et al. (2012); Rozza and Veroy (2007).

For the case of European options no dual space is required and thus the steps in Algorithm 1 involving the Lagrange multiplier space are omitted, resulting in a standard POD-Greedy algorithm.

\section{Calibration procedure}

Here we describe the procedure for the calibration problem (2.1) using the Heston model Heston (1993).

Given $S_{0}$ and the observations $P_{i}^{\text {obs }}$ at $\left(T_{i}, K_{i}\right), i=1, \ldots, M$, we denote by $P_{i}\left(\nu_{0}, S_{0}, T_{i}, K_{i}\right)$ the prices in the Heston model, where $\nu_{0} \in \mathbb{R}_{+}$is the initial volatility. Since, the value of $\nu_{0}$ is not observable on the market, it needs to be defined together with the parameters $\xi, \rho, \gamma, \kappa$. We collect all parameters to be identified into the single vector $\boldsymbol{\Theta}=$ $\left(\Theta_{1}, \ldots, \Theta_{5}\right)=\left(\xi, \rho, \gamma, \kappa, \nu_{0}\right) \in \mathcal{P}_{\text {opt }}$, where

$$
\mathcal{P}_{\text {opt }}:=\left\{\Theta \in \mathbb{R}^{5}: \Theta_{\min , i} \leq \Theta_{i} \leq \Theta_{\max , i}, \quad i=1, \ldots, 5\right\} .
$$

Then the minimization problem (2.1) for the Heston model can be written in the following form

$$
\min _{\boldsymbol{\Theta} \in \mathcal{P}_{\mathrm{opt}}} J(\boldsymbol{\Theta}), \quad J(\boldsymbol{\Theta}):=\frac{1}{M} \sum_{i=1}^{M}\left|P_{i}^{\mathrm{obs}}-P_{i}(\boldsymbol{\Theta})\right|^{2} .
$$

We apply the FEM and the RBM to compute the model prices $P_{i}(\boldsymbol{\Theta})$. For $i=1, \ldots, M$ we denote the approximate model price as

$$
P_{i}^{n, \mathrm{~s}}(\mathbf{\Theta}):=w_{n}\left(T_{i}, \log \left(S_{0} / K_{i}\right), \nu_{0} ; \boldsymbol{\mu}\right),
$$

where the superscript $n=\mathcal{N}$ corresponds to the FEM approximation and $n=N$ stands for the RBM approximation, $s=\{\mathrm{Eu}, \mathrm{Am}\}$. Here $w_{n}(\boldsymbol{\mu}):=u_{n}(\boldsymbol{\mu})+u_{L \mathcal{N}}(\boldsymbol{\mu})$ and $u_{n}(\boldsymbol{\mu})$ is a solution of $\mathbb{E}_{n}(\boldsymbol{\mu}):=\mathbb{E}_{n}^{s}(\boldsymbol{\mu}), s=\{\mathrm{Eu}, \mathrm{Am}\}, n=\{\mathcal{N}, N\}$ for $i=1, \ldots, M, \boldsymbol{\mu} \in \mathcal{P}$ and $T=\max \left(T_{i}\right)$. By abuse of notation, we often omit the index $s$ in the notation of $P_{i}^{\mathcal{N}, s}$, if it is clear from context. Then we obtain the following minimization problem for the detailed $(n=\mathcal{N})$ or reduced $(n=N)$ models

$$
\min _{\boldsymbol{\Theta} \in \mathcal{P}_{\mathrm{opt}}} J_{n}(\boldsymbol{\Theta}), \quad J_{n}(\boldsymbol{\Theta}):=\frac{1}{M} \sum_{i=1}^{M}\left|P_{i}^{\mathrm{obs}}-P_{i}^{n}(\boldsymbol{\Theta})\right|^{2} .
$$

We note that due to the presence of the box constraints this finite-dimensional minimization problem admits a solution due to continuity of the objective functional.

We also extend the de-Americanization strategy to the calibration of the Heston model. Let $\widetilde{P}_{j}^{\text {obs }}$ are the pseudo-European put prices obtained by the DAS. We consider the FEM and the semi-closed-form solutions $P_{i}^{\mathrm{CF}}$ Heston (1993) to compute the European put model prices. Then the minimization problem (3.1) becomes

$$
\min _{\boldsymbol{\Theta} \in \mathcal{P}_{\mathrm{opt}}} \widetilde{J}_{n}(\boldsymbol{\Theta}), \quad \widetilde{J}_{n}(\boldsymbol{\Theta}):=\frac{1}{M} \sum_{i=1}^{M}\left|\widetilde{P}_{i}^{\text {obs }}-P_{i}^{n}(\boldsymbol{\Theta})\right|^{2},
$$


where $P_{i}^{n}(\boldsymbol{\Theta})$ are European put prices in the Heston model computed by either the FEM $(n=\mathcal{N})$ or by the semi-closed-form solutions $(n=\mathrm{CF})$.

Remark 5.1. One could also consider a combination of the RBM with the de-Americanization strategy, i.e., applying the $R B M$ to approximate $\mathbb{E}_{\mathcal{N}}^{\mathrm{Eu}}$ by $\mathbb{E}_{N}^{\mathrm{Eu}}$. The corresponding minimization problem can be stated as follows

$$
\min _{\boldsymbol{\Theta} \in \mathcal{P}_{\mathrm{opt}}} \widetilde{J}_{N}(\boldsymbol{\Theta}), \quad \widetilde{J}_{N}(\boldsymbol{\Theta}):=\frac{1}{M} \sum_{i=1}^{M}\left|\widetilde{P}_{i}^{\text {obs }}-P_{i}^{N}(\boldsymbol{\Theta})\right|^{2},
$$

with $P_{i}^{N}(\boldsymbol{\Theta})=P_{i}^{N, \mathrm{Eu}}(\boldsymbol{\Theta})$.

Remark 5.2. Note that the interest rate $r$ is not determined by a calibration procedure and is fixed beforehand. In our case, the market data will be a single stock in the U.S., and for an approximation of the risk-free rate we use the rates of the U.S. Department of the Treasury. Hence, to construct the reduced basis spaces, we only need to consider the variation of four parameters $\boldsymbol{\mu}=(\xi, \rho, \gamma, \kappa) \in \mathcal{P} \subset \mathbb{R}^{4}$. However, this choice is restrictive and for new market data we would need to construct a new reduced basis set. Therefore, to conserve generality in our approach, we consider the variation of all parameters, $\boldsymbol{\mu}=(\xi, \rho, \gamma, \kappa, r)$, and consequently the constructed reduced basis will be entirely market-independent.

\section{NumericAl RESUlts}

Before we provide concrete numerical results, we comment on the algorithmic aspects of the optimization procedure. We first note, that the problems under consideration belong to the class of finite-dimensional optimization problems with box constraints. For the numerical realization, we use the MATLAB Optimization Toolbox and apply the built-in optimization solver lsqnonlin or fmincon, in which the gradients of the prices with respect to the parameters are approximated by finite differences.

We note that, for the case of American put options, due to the presence of the inequality constraints in the option pricing model, the objective functional may not be differentiable at certain parameter values, see, e.g., Achdou and Pironneau (2005a). However, problems with differentiability are rarely encountered in practical computations, cf. also (Achdou and Pironneau, 2005a, Remark 5).

For the numerical experiments we set $T=2, I=250, \Delta t=T / I=0.008, \theta=1 / 2$. The computational domain $\Omega=\left(\nu_{\min }, \nu_{\max }\right) \times\left(x_{\min }, x_{\max }\right)=\left(10^{-5}, 3\right) \times(-5,5)$ is resolved by a triangulation $\mathcal{T}_{\mathcal{N}}$ of $\Omega$, consisting of $J$ simplices $T_{\mathcal{N}}^{j}, 1 \leq j \leq J$, such that $\bar{\Omega}=\cup_{T_{\mathcal{N}} \in \mathcal{T}_{\mathcal{N}}} \bar{T}_{\mathcal{N}}$. We use standard conforming nodal first-order finite element approximation spaces $X_{\mathcal{N}} \subset X$, $V_{\mathcal{N}} \subset V$, where $X_{\mathcal{N}}:=\left\{v \in X: \quad v_{\mid T_{\mathcal{N}}^{j}} \in \mathbb{P}^{1}\left(T_{\mathcal{N}}^{j}\right), 1 \leq j \leq J\right\}$, and $\mathbb{P}^{1}$ is a space of linear polynomials with degree at most one, and $\operatorname{dim}\left(X_{\mathcal{N}}\right)=\mathcal{N}_{X}=4753$. To discretize the dual space $V^{\prime}$, we use discontinuous piecewise linear biorthogonal basis functions defined on the same mesh as the basis functions of $V_{\mathcal{N}}$, Wohlmuth (2000). That is, $V_{\mathcal{N}}^{\prime}:=\operatorname{span}\left\{\zeta_{q}, q=\right.$ $\left.1, \ldots, \mathcal{N}_{V^{\prime}}\right\}, \operatorname{dim}\left(V_{\mathcal{N}}^{\prime}\right)=\mathcal{N}_{V^{\prime}}=\mathcal{N}_{V}$, where $\zeta_{q}$ satisfy a local biorthogonality relation:

$$
\int_{T_{\mathcal{N}}^{j}} \zeta_{q} \phi_{p}=\delta_{p q} \int_{T_{\mathcal{N}}^{j}} \phi_{p} \geq 0, \quad \phi_{p} \in V_{\mathcal{N}}, \quad p, q=1, \ldots, \mathcal{N}_{V}
$$

For these settings of the discrete spaces, we obtain that $\left(V_{\mathcal{N}}, V_{\mathcal{N}}^{\prime}\right)$ form a uniformly inf-sup stable pairing. 
For $\boldsymbol{\mu}:=(\xi, \rho, \gamma, \kappa, r) \in \mathcal{P} \subset \mathbb{R}^{5}$ and $\boldsymbol{\Theta}:=\left(\xi, \rho, \gamma, \kappa, \nu_{0}\right) \in \mathcal{P}^{\text {opt }} \subset \mathbb{R}^{5}$, we define

$$
\begin{aligned}
\mathcal{P} & \equiv[0.1,0.9] \times[-0.95,0.95] \times[0.01,0.5] \times[0.1,5] \times[0.0001,0.8] \\
\mathcal{P}^{\mathrm{opt}} & \equiv[0.1,0.9] \times[-0.95,0.3] \times[0.01,0.5] \times[0.1,5] \times\left[10^{-5}, 1\right] .
\end{aligned}
$$

We set $\nu_{\min }=10^{-5}$ to be sufficiently small, compared to the range of the possible values of the volatility $\nu$, in order to reduce the effect of the truncation of the boundary on the option price for small values of $\nu$. Unless otherwise stated, the calibration routine is performed with lsqnonlin, which uses a Trust-Region-Reflective algorithm, and the stopping criterion is set as $J(\boldsymbol{\Theta})-J\left(\boldsymbol{\Theta}^{\star}\right) \leq 10^{-12},\left\|\boldsymbol{\Theta}-\boldsymbol{\Theta}^{\star}\right\|_{2} \leq 10^{-5}$, where $\boldsymbol{\Theta}^{\star}$ is a locally optimal solution. The algebraic system of equations resulting from the detailed $\mathbb{E}_{\mathcal{N}}^{\mathrm{Am}}(\boldsymbol{\mu})$ and reduced $\mathbb{E}_{N}^{\mathrm{Am}}(\boldsymbol{\mu})$ problems are solved using the Primal-Dual-Active-Set strategy.

6.1. Calibration based on RBM. We consider a training set $\mathcal{P}_{\text {train }}$ composed of uniformly distributed points in $\mathcal{P}$ with $\left|\mathcal{P}_{\text {train }}\right|=1024$ for the European put and $\left|\mathcal{P}_{\text {train }}\right|=3125$ for the American put options. The bases are generated by the POD-Greedy and PODAngle-Greedy algorithms for the European and the American options, respectively. The reduced systems have dimension $N_{\max }=100$ for European put and $N_{\max }=125$ for American put options. Firstly, we consider the quality of the calibration in terms of the RBM applied to a synthetic data set with $r=5 \%$ :

$$
\begin{array}{ll}
S_{0}=1, & \\
T_{1}=\frac{1}{6}, & K_{1}=\{0.95,0.975,1,1.025,1.05\}, \\
T_{2}=\frac{1}{2}, & K_{2}=K_{1} \cup\{0.9,0.925,1.075,1.1\}, \\
T_{3}=\frac{3}{4}, & K_{3}=K_{2} \cup\{0.85,0.875,1.125,1.15\}, \\
T_{4}=1, & K_{4}=K_{3} \cup\{0.8,0.825,1.175,1.2\}, \\
T_{5}=2, & K_{5}=K_{4} \cup\{0.75,0.775,1.225,1.25\} .
\end{array}
$$

For each pair $\left(T_{i}, K_{i}\right)_{i=1, \ldots, 5}$, we generate two artificial sets of observations $P^{\text {obs }}$ consisting of 65 European and American put options at $\boldsymbol{\Theta}=(0.7,-0.8,0.3,1.4,0.3)$. That is, we solve the detailed problems $\mathbb{E}_{\mathcal{N}}^{\mathrm{Am}}$ and $\mathbb{E}_{\mathcal{N}}^{\mathrm{Eu}}$ for the parameter $\boldsymbol{\mu}=(0.7,-0.8,0.3,1.4,0.05)$ and $K=1$ and interpolate the corresponding solution $K_{i} u_{\mathcal{N}}\left(T_{i}, \nu, x ; \boldsymbol{\mu}\right)$ at $\nu^{\star}=\nu_{0}, x_{i}^{\star}=$ $\log \left(S_{0} / K_{i}\right)$.

We perform the optimization routine (5.3) with the reduced surrogate model $(n=N)$ and the high-fidelity detailed problem $(n=\mathcal{N})$. In both cases, we use the same initial guess $\Theta_{\text {in }}$ for the optimization algorithm. The results of the calibration for two different data sets of American and European options are presented in Table 1. We observe that, using the detailed models $\mathbb{E}_{\mathcal{N}}^{s},\{s=\mathrm{Am}, \mathrm{Eu}\}$, we can basically recover the exact parameters $\boldsymbol{\Theta}_{\text {ex }}$. The reduced surrogate models provide still accurate enough results but are computationally much less expensive.

The influence of the calibration process on the accuracy of the option price for different strike and maturity values are shown in Figure 2 for both American and European options. In all plots, we hardly observe any differences in the option price obtained from the synthetic and the calibrated data with the detailed problem and the reduced problem.

The run-time performance is reported in Table 2. Additionally, we state the number of required iteration steps in the optimization algorithm and the number of function calls. 


\begin{tabular}{ccccccccc}
\hline Method & $\mathbb{E}(\boldsymbol{\mu})$ & $\boldsymbol{\Theta}$ & $\xi$ & $\rho$ & $\gamma$ & $\kappa$ & $\nu_{0}$ & $\left\|\boldsymbol{\Theta}_{\text {ex }}-\boldsymbol{\Theta}^{\star}\right\|_{2}$ \\
\hline & & $\boldsymbol{\Theta}_{\text {ex }}$ & 0.700 & -0.800 & 0.300 & 1.400 & 0.300 & \\
& & $\boldsymbol{\Theta}_{\text {in }}$ & 0.601 & -0.682 & 0.487 & 2.020 & 0.496 & \\
\hline$J_{\mathcal{N}}(\boldsymbol{\Theta})$ & $\mathbb{E}_{\mathcal{N}}^{\mathrm{Am}}$ & $\boldsymbol{\Theta}^{\star}$ & 0.700 & -0.800 & 0.300 & 1.399 & 0.300 & $2.14 \mathrm{e}-5$ \\
$J_{N}(\boldsymbol{\Theta})$ & $\mathbb{E}_{N}^{\mathrm{Am}}$ & $\boldsymbol{\Theta}^{\star}$ & 0.694 & -0.831 & 0.298 & 1.447 & 0.303 & $5.62 \mathrm{e}-2$ \\
$J_{\mathcal{N}}(\boldsymbol{\Theta})$ & $\mathbb{E}_{\mathcal{N}}^{\mathrm{Eu}}$ & $\boldsymbol{\Theta}^{\star}$ & 0.700 & -0.800 & 0.300 & 1.399 & 0.300 & $2.05 \mathrm{e}-5$ \\
$J_{N}(\boldsymbol{\Theta})$ & $\mathbb{E}_{N}^{\mathrm{Eu}}$ & $\boldsymbol{\Theta}^{\star}$ & 0.616 & -0.886 & 0.293 & 1.306 & 0.300 & $1.52 \mathrm{e}-1$ \\
\hline
\end{tabular}

TABLE 1. Calibration of the synthetic data set of American and European put options in the Heston model using detailed and reduced problems.
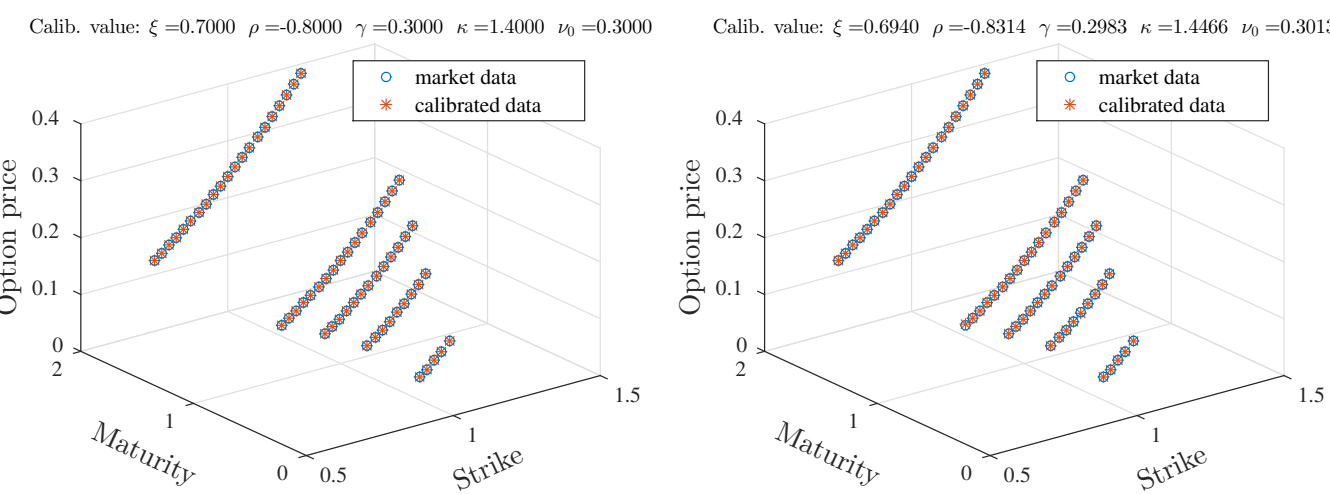

Calib. value: $\xi=0.7000 \quad \rho=-0.8000 \quad \gamma=0.3000 \quad \kappa=1.4000 \quad \nu_{0}=0.3000$

Calib. value: $\xi=0.6161 \quad \rho=-0.8859 \quad \gamma=0.2926 \quad \kappa=1.3062 \quad \nu_{0}=0.3003$
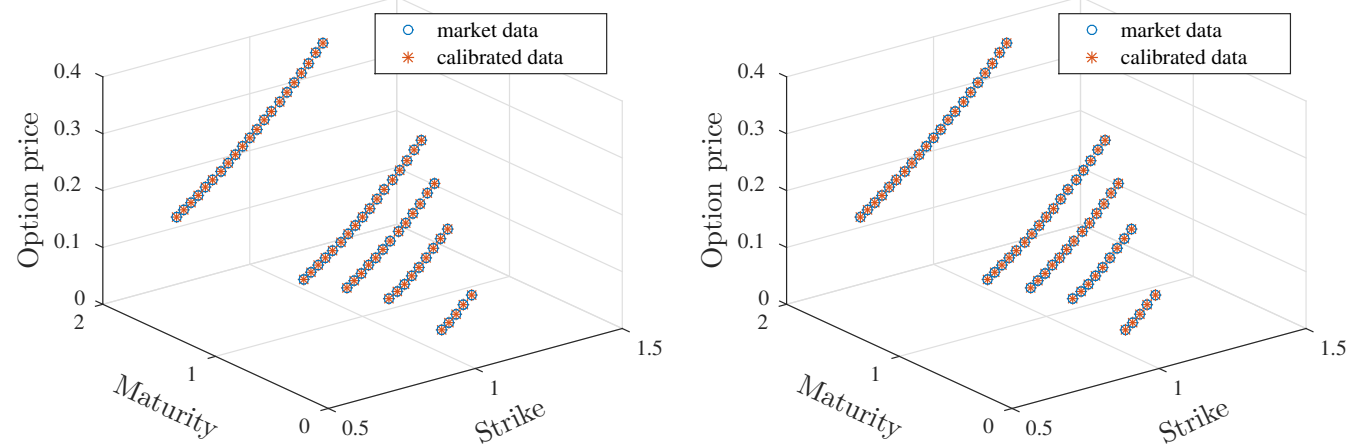

FiguRE 2. Results of the calibration to the synthetic data set of American (upper row) and European (lower row) put options in the Heston model using the detailed model $\mathbb{E}_{\mathcal{N}}(\boldsymbol{\mu})$ (left) and the reduced surrogate model $\mathbb{E}_{N}(\boldsymbol{\mu})$ (right). The circles are the synthetic prices and the stars are the prices in the calibrated model.

In this example, the optimization routine with the surrogate reduced model is about 100 times faster for American put options and about 350 times faster for European put options. The reduced model for American options recovers the parameter slightly better than the European one. This fact can be explained by the larger dimension of the reduced system for American options and the larger training set, which is also reflected in the run-time performance. We point out, that depending on the priority of the task, i.e., accuracy vs. the run-time, one can always manually adjust the dimension of the reduced system. 


\begin{tabular}{cccccc}
\hline Method & $\mathbb{E}(\boldsymbol{\mu})$ & \# iter. & $\# J$ & calib. time & $J\left(\boldsymbol{\Theta}^{\star}\right)$ \\
\hline$J_{\mathcal{N}}(\boldsymbol{\Theta})$ & $\mathbb{E}_{\mathcal{N}}^{\mathrm{Am}}$ & 7 & 48 & $15.59 \mathrm{hrs}$ & $1.698 \mathrm{e}-16$ \\
$J_{N}(\boldsymbol{\Theta})$ & $\mathbb{E}_{N}^{\mathrm{Am}}$ & 8 & 54 & $9.50 \mathrm{~min}$ & $9.515 \mathrm{e}-9$ \\
$J_{\mathcal{N}}(\boldsymbol{\Theta})$ & $\mathbb{E}_{\mathcal{N}}^{\mathrm{Eu}}$ & 7 & 48 & $11.67 \mathrm{hrs}$ & $1.242 \mathrm{e}-16$ \\
$J_{N}(\boldsymbol{\Theta})$ & $\mathbb{E}_{N}^{\mathrm{Eu}}$ & 11 & 72 & $1.996 \mathrm{~min}$ & $1.157 \mathrm{e}-08$ \\
\hline
\end{tabular}

TABLE 2. Calibration results for the synthetic data set of American and European put options in the Heston model in terms of the run-time performance. The number "\# iter." corresponds to the number of iterations and "\# $J$ " is the total number of function evaluations performed by the optimization routine.

To quantify the differences between reduced and detailed calibration, we plot the pointwise relative errors $\left|P_{i}^{\text {obs }}-P_{i}^{s}\left(\Theta^{\star}\right)\right| / P_{i}^{\text {obs }}, s=\{\mathcal{N}, N\}, i=1, \ldots, M$ in Figure 3 . We observe that the reduced models yield a very good fit to the synthetic data with relative errors within the $0.5 \%$-margin.
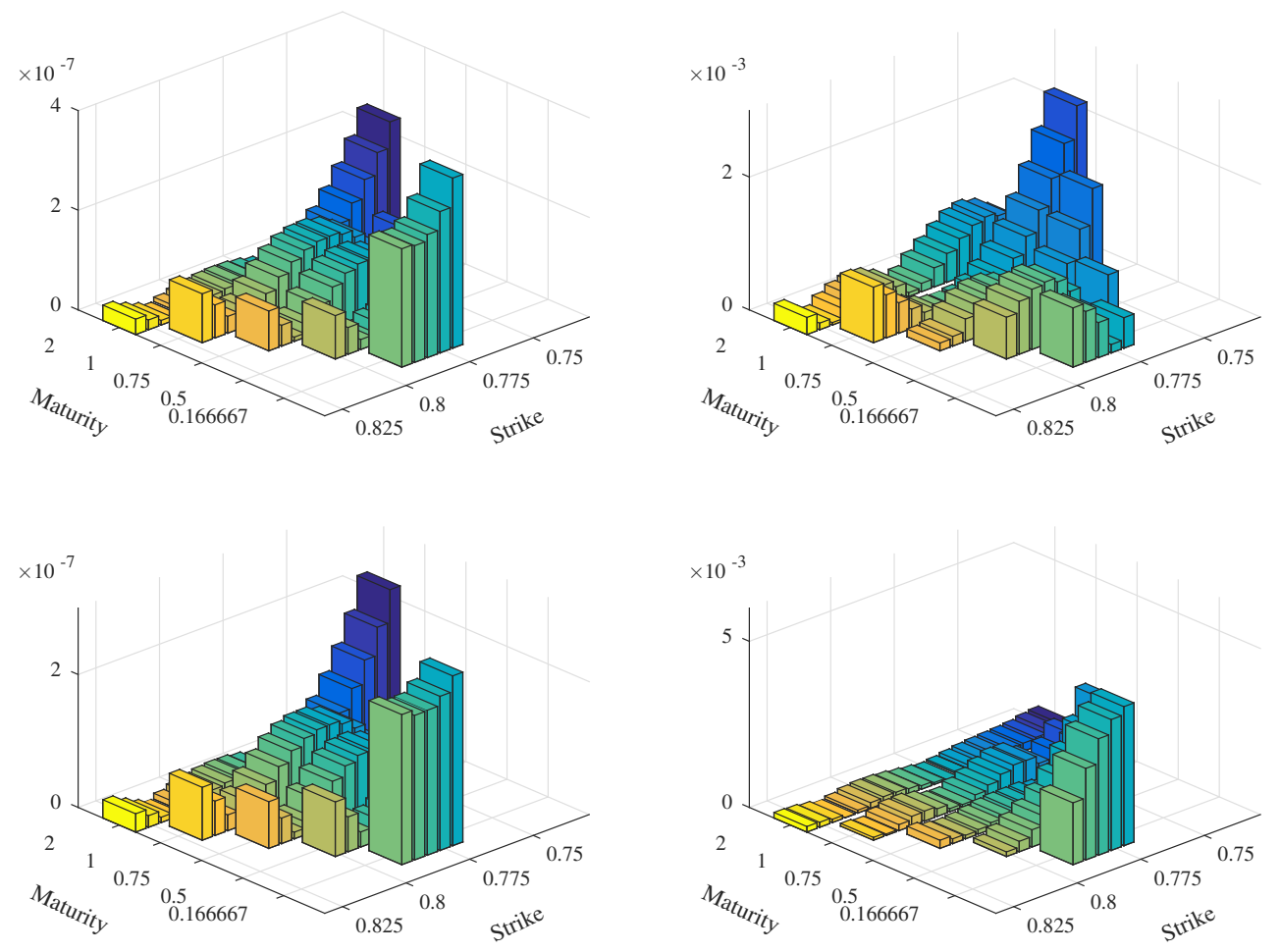

Figure 3. Calibration results for the synthetic data set of American (upper row) and European (lower row) put options in terms of point-wise absolute relative errors, $\left|P_{i}^{\text {obs }}-P_{i}^{s, \mathrm{Am}}\left(\boldsymbol{\Theta}^{\star}\right)\right| / P_{i}^{\text {obs }}, i=1, \ldots, M$, using the Heston model. Left: calibration with the detailed model $\mathbb{E}_{\mathcal{N}}(\boldsymbol{\mu}), s=\mathcal{N}$. Right: calibration with the reduced surrogate model $\mathbb{E}_{N}(\boldsymbol{\mu}), s=N$. 
6.2. Comparison between RBM and DAS. Next, we perform a numerical comparison of the calibration with American put options using both model reduction techniques. That is, we consider the minimization problems $(5.3)(n=N)$ and $(5.4)(n=\mathcal{N})$. For comparative purposes, we also carry out the calibration with the detailed finite element solution (5.4) $(n=\mathcal{N})$.

First, we use a synthetic set of observations $P^{\text {obs }}$ given by (6.3). We consider different parameter scenarios corresponding to different values of $\boldsymbol{\Theta} \in \mathcal{P}_{\text {opt }}$; see Table 3. For each scenario, we construct an artificial set of observations $P_{i}^{\text {obs }}:=P_{i}^{\mathcal{N}, A m}, i=1, \ldots, 65$. In general, the parameter $\kappa$ is price-insensitive, see, e.g., Janek et al. (2011), and thus it cannot be reconstructed. Therefore, we fix $\kappa$ to its exact value and do the parameter estimation only for $\xi, \rho, \gamma$, and $\nu_{0}$. The results of the calibration are summarized in Tables 4 and 5 . We observe that, overall, all methods provide a good reconstruction of the parameters. As can be expected, the most cost-intense variant also provides the results with the highest accuracy. However all of our surrogate models yield quite good results.

\begin{tabular}{cccccc}
\hline & $\xi$ & $\rho$ & $\gamma$ & $\kappa$ & $\nu_{0}$ \\
\hline$p_{1}$ & 0.10 & -0.20 & 0.07 & 0.5 & 0.07 \\
$p_{2}$ & 0.25 & -0.50 & 0.10 & 0.5 & 0.10 \\
$p_{3}$ & 0.40 & -0.50 & 0.15 & 0.6 & 0.15 \\
$p_{4}$ & 0.55 & -0.45 & 0.20 & 1.2 & 0.20 \\
$p_{5}$ & 0.70 & -0.80 & 0.30 & 1.4 & 0.30 \\
$p_{6}$ & 0.2928 & -0.7571 & 0.0707 & 0.6067 & 0.0707
\end{tabular}

TABLE 3. Overview of the parameter sets used to generate the synthetic data set.

We observe that both reduction approaches provide a significant speed-up compared to the expensive detailed solver, which on average takes about eight hours for each scenario. Although the DAS allows for an extremely efficient calibration process, it requires an additional pre-processing step for the data. In contrast to the RBM, this pre-processing depends on the actual market data and therefore can not be performed in advance. This is a serious bottleneck compared to the RBM approach.

Figure 4 shows the influence of the different calibration approaches on the parameters. It can be seen that in all approaches the main difficulty arises in in identifying $\xi$ and $\rho$. In fact, this tendency has been also observed for the detailed solver (see cases $p_{1}, p_{4}$, Table 5 ). The remaining parameters $\gamma$ and $\nu_{0}$ are recovered almost exactly. We also note that for scenarios $p_{1}-p_{3}$, which correspond to the cases when $\xi, \nu_{0}$ and $\rho$ are the smallest, the DAS calibration is able to provide a better reconstruction of the parameter $\xi$ than the RBM. By contrast, in the scenarios $p_{4}$ and $p_{5}$, which correspond to large values of the correlation parameter, the DAS gives poorer results for $\xi$ and $\rho$.

To summarize our findings, the cases with "extreme" parameter values have a significant impact on the performance of the optimization routine, in both the detailed and the reduced problems. A numerical study of the effect of the de-Americanization method for such "extreme" scenarios has been investigated in great details in a recent work Burkovska et al. (2018). In the case of the reduced basis method, this difficulty may be overcome for example by increasing the number of snapshots or increasing the dimension of the reduced system. This, of course, may result in the computational increase of the offline cost to construct the reduced bases and of the online cost required to compute the solution. 


\begin{tabular}{|c|c|c|c|c|c|}
\hline Scenario & Method & $\mathbb{E}(\boldsymbol{\mu})$ & $\# J$ & calib. time & pre-process. time for $P^{\text {obs }}$ \\
\hline$p_{1}$ & $\begin{array}{l}J_{\mathcal{N}}(\boldsymbol{\Theta}) \\
\widetilde{J}_{\mathcal{N}}(\boldsymbol{\Theta}) \\
J_{N}(\boldsymbol{\Theta}) \\
\end{array}$ & $\begin{array}{l}\mathbb{E}_{\mathcal{N}}^{\mathrm{Am}} \\
\mathbb{E}_{\mathcal{N}}^{\mathrm{Eu}} \\
\mathbb{E}_{N}^{\mathrm{Am}} \\
\end{array}$ & $\begin{array}{c}75 \\
145 \\
70 \\
\end{array}$ & $\begin{array}{c}8.524 \mathrm{hrs} \\
18.018 \mathrm{~min} \\
3.912 \mathrm{~min}\end{array}$ & $36.045 \mathrm{~min}$ \\
\hline$p_{2}$ & $\begin{array}{l}J_{\mathcal{N}}(\boldsymbol{\Theta}) \\
\widetilde{J}_{\mathcal{N}}(\boldsymbol{\Theta}) \\
J_{N}(\boldsymbol{\Theta}) \\
\end{array}$ & $\begin{array}{l}\mathbb{E}_{\mathcal{N}}^{\mathrm{Am}} \\
\mathbb{E}_{\mathcal{N}}^{\mathrm{Eu}} \\
\mathbb{E}_{N}^{\mathrm{Am}} \\
\end{array}$ & $\begin{array}{l}65 \\
70 \\
70 \\
\end{array}$ & $\begin{array}{l}8.035 \mathrm{hrs} \\
8.895 \mathrm{~min} \\
3.489 \mathrm{~min} \\
\end{array}$ & $36.830 \mathrm{~min}$ \\
\hline$p_{3}$ & $\begin{array}{l}J_{\mathcal{N}}(\boldsymbol{\Theta}) \\
\widetilde{J}_{\mathcal{N}}(\boldsymbol{\Theta}) \\
J_{N}(\boldsymbol{\Theta})\end{array}$ & $\begin{array}{l}\mathbb{E}_{\mathcal{N}}^{\mathrm{Am}} \\
\mathbb{E}_{\mathcal{N}}^{\mathrm{Eu}} \\
\mathbb{E}_{N}^{\mathrm{Am}}\end{array}$ & $\begin{array}{l}60 \\
60 \\
70 \\
\end{array}$ & $\begin{array}{l}8.341 \mathrm{hrs} \\
8.083 \mathrm{~min} \\
3.574 \mathrm{~min}\end{array}$ & $35.374 \mathrm{~min}$ \\
\hline$p_{4}$ & $\begin{array}{l}J_{\mathcal{N}}(\boldsymbol{\Theta}) \\
\widetilde{J}_{\mathcal{N}}(\boldsymbol{\Theta}) \\
J_{N}(\boldsymbol{\Theta})\end{array}$ & $\begin{array}{l}\mathbb{E}_{\mathcal{N}}^{\mathrm{Am}} \\
\mathbb{E}_{\mathcal{N}}^{\mathrm{Eu}} \\
\mathbb{E}_{N}^{\mathrm{Am}}\end{array}$ & $\begin{array}{l}50 \\
45 \\
55\end{array}$ & $\begin{array}{l}7.088 \mathrm{hrs} \\
6.031 \mathrm{~min} \\
2.813 \mathrm{~min}\end{array}$ & $36.660 \mathrm{~min}$ \\
\hline$p_{5}$ & $\begin{array}{l}J_{\mathcal{N}}(\boldsymbol{\Theta}) \\
\widetilde{J}_{\mathcal{N}}(\boldsymbol{\Theta}) \\
J_{N}(\boldsymbol{\Theta})\end{array}$ & $\begin{array}{l}\mathbb{E}_{\mathcal{N}}^{\mathrm{Am}} \\
\mathbb{E}_{\mathcal{N}}^{\mathrm{Eu}} \\
\mathbb{E}_{N}^{\mathrm{Am}}\end{array}$ & $\begin{array}{l}40 \\
65 \\
45\end{array}$ & $\begin{array}{l}6.331 \mathrm{hrs} \\
8.607 \mathrm{~min} \\
2.271 \mathrm{~min}\end{array}$ & $36.574 \mathrm{~min}$ \\
\hline$p_{6}$ & $\begin{array}{l}J_{\mathcal{N}}(\boldsymbol{\Theta}) \\
\widetilde{J}_{\mathcal{N}}(\boldsymbol{\Theta}) \\
J_{N}(\boldsymbol{\Theta})\end{array}$ & $\begin{array}{l}\mathbb{E}_{\mathcal{N}}^{\mathrm{Am}} \\
\mathbb{E}_{\mathcal{N}}^{\mathrm{Eu}} \\
\mathbb{E}_{N}^{\mathrm{Am}}\end{array}$ & $\begin{array}{l}70 \\
70 \\
90\end{array}$ & $\begin{array}{l}9.665 \mathrm{hrs} \\
9.555 \mathrm{~min} \\
4.503 \mathrm{~min}\end{array}$ & $36.960 \mathrm{~min}$ \\
\hline
\end{tabular}

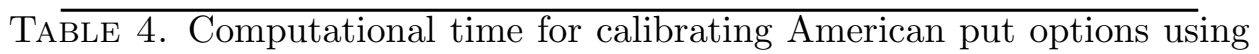
different model reduction techniques.

However, in contrast to the DAS, the theoretical framework of the RBM allows to derive efficient a posteriori error estimates which can be used to speed up the construction of the reduced model as well as to certify the approximation quality and reliability of the method.

6.3. Real market data. Finally, we extend our approach to the calibration of a real market data set, provided by options on the Google stock. Since the Google stock does not pay dividends, the American call options can be priced the same as the European call options, Hull (2003). Hence, we restrict consideration to only American put options. Namely, we consider the data $P^{\text {obs }}$ of 401 American put options with $S_{0}=523.755, r=$ $0.15 \%$ on February 2nd, 2015. The data is pre-processed using the methodology applied to the volatility index (VIX) by the Chicago board of exchange CBOE (2009):

- For each option with strike price $K_{i}$, we consider the midpoint of the bid-ask spread.

- Options with zero bid prices are neglected.

- If two puts with consecutive strike prices have zero bid prices, no puts with lower strike prices are included.

The used data is given in Appendix A, see Table A.1. In terms of the moneyness, we consider all types, out-of-the money $\left(K_{i}<S_{0}\right)$, at-the-money $\left(K_{i}=S_{0}\right)$ and in-the-money $\left(K_{i}>S_{0}\right)$ options.

In our synthetic test scenarios, the Feller condition (2.3) was automatically satisfied. However, this does not hold for general calibration processes. Thus, we impose the following additional constraint on $\boldsymbol{\Theta}=\left(\xi, \rho, \gamma, \kappa, \nu_{0}\right) \in \mathcal{P}_{\text {opt }}$

$$
\mathcal{P}_{\text {opt }}:=\left\{\Theta \in \mathbb{R}^{5}: \Theta_{\min , i} \leq \Theta_{i} \leq \Theta_{\max , i}, \quad 2 \Theta_{3} \Theta_{4}-\Theta_{1}^{2}<0, \quad i=1, \ldots, 5\right\} .
$$




\begin{tabular}{|c|c|c|c|c|c|c|c|c|}
\hline Scenario & Method & $\mathbb{E}(\boldsymbol{\mu})$ & $\Theta$ & $\xi$ & $\rho$ & $\gamma$ & $\nu_{0}$ & $J\left(\boldsymbol{\Theta}^{\star}\right)$ \\
\hline$p_{1}$ & $\begin{array}{l}J_{\mathcal{N}}(\boldsymbol{\Theta}) \\
\widetilde{J}_{\mathcal{N}}(\boldsymbol{\Theta}) \\
J_{N}(\boldsymbol{\Theta})\end{array}$ & $\begin{array}{l}\mathbb{E}_{\mathcal{N}}^{\mathrm{Am}} \\
\mathbb{E}_{\mathcal{N}}^{\mathrm{Eu}} \\
\mathbb{E}_{N}^{\mathrm{Am}} \\
\end{array}$ & $\begin{array}{l}\Theta_{\text {ex }} \\
\Theta^{\star} \\
\Theta^{\star} \\
\Theta^{\star}\end{array}$ & $\begin{array}{l}0.1 \\
0.1002 \\
0.1000 \\
0.1477\end{array}$ & $\begin{array}{l}-0.2 \\
-0.1997 \\
-0.3003 \\
-0.0788\end{array}$ & $\begin{array}{l}0.07 \\
0.07 \\
0.0701 \\
0.0697\end{array}$ & $\begin{array}{l}0.07 \\
0.07 \\
0.0688 \\
0.0700\end{array}$ & $\begin{array}{l}7.8806 \mathrm{e}-14 \\
1.0248 \mathrm{e}-07 \\
4.3440 \mathrm{e}-08\end{array}$ \\
\hline$p_{2}$ & $\begin{array}{l}J_{\mathcal{N}}(\boldsymbol{\Theta}) \\
\widetilde{J}_{\mathcal{N}}(\boldsymbol{\Theta}) \\
J_{N}(\boldsymbol{\Theta})\end{array}$ & $\begin{array}{l}\mathbb{E}_{\mathcal{N}}^{\mathrm{Am}} \\
\mathbb{E}_{\mathcal{N}}^{\mathrm{Eu}} \\
\mathbb{E}_{N}^{\mathrm{Am}}\end{array}$ & $\begin{array}{l}\Theta_{\text {ex }} \\
\Theta^{\star} \\
\Theta^{\star} \\
\Theta^{\star}\end{array}$ & $\begin{array}{l}0.25 \\
0.25 \\
0.2404 \\
0.2860\end{array}$ & $\begin{array}{l}-0.5 \\
-0.5 \\
-0.5388 \\
-0.4824\end{array}$ & $\begin{array}{l}0.1 \\
0.1 \\
0.1001 \\
0.0968\end{array}$ & $\begin{array}{l}0.1 \\
0.1 \\
0.0991 \\
0.1015\end{array}$ & $\begin{array}{l}6.4756 \mathrm{e}-17 \\
1.1363 \mathrm{e}-08 \\
9.9148 \mathrm{e}-08\end{array}$ \\
\hline$p_{3}$ & $\begin{array}{l}J_{\mathcal{N}}(\boldsymbol{\Theta}) \\
\widetilde{J}_{\mathcal{N}}(\boldsymbol{\Theta}) \\
J_{N}(\boldsymbol{\Theta})\end{array}$ & $\begin{array}{l}\mathbb{E}_{\mathcal{N}}^{\mathrm{Am}} \\
\mathbb{E}_{\mathcal{N}}^{\mathrm{Eu}} \\
\mathbb{E}_{N}^{\mathrm{Am}}\end{array}$ & $\begin{array}{l}\Theta_{e x} \\
\Theta^{\star} \\
\Theta^{\star} \\
\Theta^{\star}\end{array}$ & $\begin{array}{l}0.4 \\
0.4 \\
0.4282 \\
0.3537\end{array}$ & $\begin{array}{l}-0.5 \\
-0.5 \\
-0.4620 \\
-0.5731\end{array}$ & $\begin{array}{l}0.15 \\
0.15 \\
0.1544 \\
0.1456\end{array}$ & $\begin{array}{l}0.15 \\
0.15 \\
0.1492 \\
0.1504\end{array}$ & $\begin{array}{l}2.9834 \mathrm{e}-18 \\
2.2003 \mathrm{e}-09 \\
6.2085 \mathrm{e}-09\end{array}$ \\
\hline$p_{4}$ & $\begin{array}{l}J_{\mathcal{N}}(\boldsymbol{\Theta}) \\
\widetilde{J}_{\mathcal{N}}(\boldsymbol{\Theta}) \\
J_{N}(\boldsymbol{\Theta})\end{array}$ & $\begin{array}{l}\mathbb{E}_{\mathcal{N}}^{\mathrm{Am}} \\
\mathbb{E}_{\mathcal{N}}^{\mathrm{Eu}} \\
\mathbb{E}_{N}^{\mathrm{Am}}\end{array}$ & $\begin{array}{l}\Theta_{\text {ex }} \\
\Theta^{\star} \\
\Theta^{\star} \\
\Theta^{\star}\end{array}$ & $\begin{array}{l}0.55 \\
0.5502 \\
0.5801 \\
0.5048\end{array}$ & $\begin{array}{l}-0.45 \\
-0.4499 \\
-0.4220 \\
-0.4980\end{array}$ & $\begin{array}{l}0.2 \\
0.2 \\
0.2044 \\
0.1989\end{array}$ & $\begin{array}{l}0.2 \\
0.2 \\
0.1989 \\
0.1995\end{array}$ & $\begin{array}{l}4.8235 \mathrm{e}-14 \\
1.5377 \mathrm{e}-09 \\
1.6681 \mathrm{e}-08\end{array}$ \\
\hline$p_{5}$ & $\begin{array}{l}J_{\mathcal{N}}(\boldsymbol{\Theta}) \\
\widetilde{J}_{\mathcal{N}}(\boldsymbol{\Theta}) \\
J_{N}(\boldsymbol{\Theta})\end{array}$ & $\begin{array}{l}\mathbb{E}_{\mathcal{N}}^{\mathrm{Am}} \\
\mathbb{E}_{\mathcal{N}}^{\mathrm{Eu}} \\
\mathbb{E}_{N}^{\mathrm{Am}}\end{array}$ & $\begin{array}{l}\Theta_{e x} \\
\Theta^{\star} \\
\Theta^{\star} \\
\Theta^{\star}\end{array}$ & $\begin{array}{l}0.7 \\
0.7 \\
0.8433 \\
0.6881\end{array}$ & $\begin{array}{l}-0.8 \\
-0.8 \\
-0.6668 \\
-0.8259\end{array}$ & $\begin{array}{l}0.3 \\
0.3 \\
0.3170 \\
0.2994\end{array}$ & $\begin{array}{l}0.3 \\
0.3 \\
0.2990 \\
0.3006\end{array}$ & $\begin{array}{l}3.4388 \mathrm{e}-18 \\
1.8369 \mathrm{e}-08 \\
1.0533 \mathrm{e}-08\end{array}$ \\
\hline$p_{6}$ & $\begin{array}{l}J_{\mathcal{N}}(\boldsymbol{\Theta}) \\
\widetilde{J}_{\mathcal{N}}(\boldsymbol{\Theta}) \\
J_{N}(\boldsymbol{\Theta})\end{array}$ & $\begin{array}{l}\mathbb{E}_{\mathcal{N}}^{\mathrm{Am}} \\
\mathbb{E}_{\mathcal{N}}^{\mathrm{Eu}} \\
\mathbb{E}_{N}^{\mathrm{Am}}\end{array}$ & $\begin{array}{l}\Theta_{e x} \\
\Theta^{\star} \\
\Theta^{\star} \\
\Theta^{\star}\end{array}$ & $\begin{array}{l}0.2928 \\
0.2928 \\
0.3690 \\
0.3096\end{array}$ & $\begin{array}{l}-0.7571 \\
0.7571 \\
-0.6026 \\
-0.7049\end{array}$ & $\begin{array}{l}0.0707 \\
0.0707 \\
0.0736 \\
0.0700\end{array}$ & $\begin{array}{l}0.0707 \\
0.0707 \\
0.0685 \\
0.0718\end{array}$ & $\begin{array}{l}6.5746 \mathrm{e}-18 \\
1.6179 \mathrm{e}-07 \\
9.6369 \mathrm{e}-08\end{array}$ \\
\hline
\end{tabular}

TABLE 5. Calibration results on the synthetic data set of American put options in the Heston model with different model reduction techniques: the RBM, the de-Americanization method and the detailed problem.

As optimization algorithm, we take the MATLAB function fmincon based on the InteriorPoint method and which, in contrast to lsqnonlin, allows the inclusion of inequality constraints. We consider the same termination condition for the optimization routine as previously.

To calibrate the parameters, we consider the detailed and reduced minimization problems (5.3) $(n=\mathcal{N}, N)$ and the DAS $(5.4)(n=\mathcal{N})$. For completeness, we also consider the calibration of the de-Americanized data using the closed-form solution of (5.4) ( $n=\mathrm{CF})$.

The results of the calibration are presented in Table 6 . As before, $\gamma$ and $\nu_{0}$ can be easily identified by all our approaches. The rate of mean reversion $\kappa$ appears to be a nonidentifiable parameter, and all models provide quite different results. For the remaining parameters $\xi$ and $\rho$, we observe that the DAS tends to underestimate the volatility of volatility $\xi$ and the correlation $\rho$, compared to the detailed and RBM approach. This is clearly reflected in all models that use the perturbed de-Americanized data, i.e., $\widetilde{J}_{\mathcal{N}}(\Theta)$ and $\widetilde{J}_{\mathrm{CF}}(\boldsymbol{\Theta})$. This is in good agreement with our observations for the synthetic data sets (see scenario $p_{5}$ and $p_{6}$ ), wherefor large (absolute) values of the correlation parameters the DAS was unable to provide a good reconstruction of the parameters $\xi$ and $\rho$. 

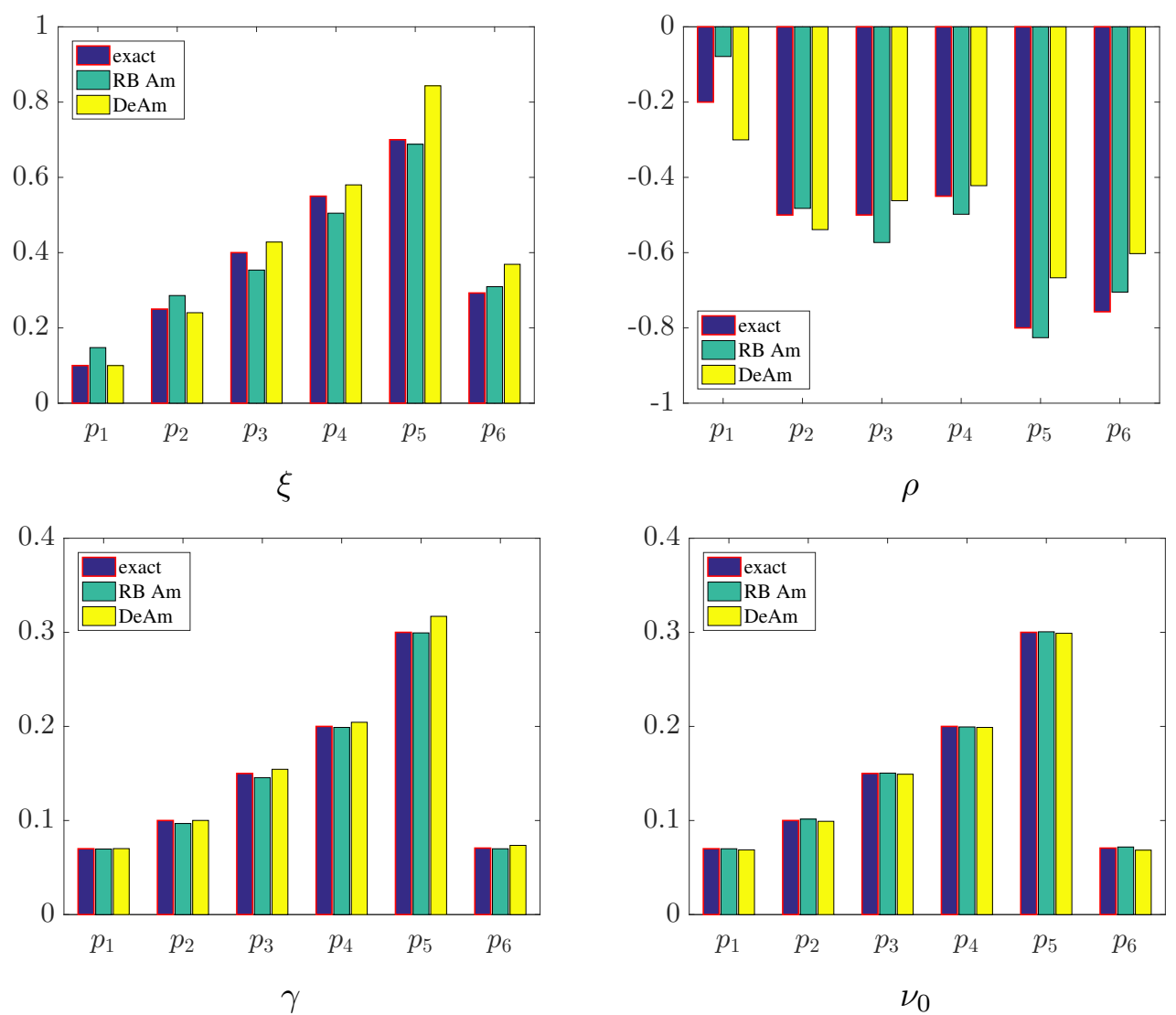

FIGURE 4. Reconstructed parameters for the different scenarios obtained by calibrating American put options with different model reduction techniques.

\begin{tabular}{cccccccc}
\hline Method & $\mathbb{E}(\boldsymbol{\mu})$ & $\boldsymbol{\Theta}$ & $\xi$ & $\rho$ & $\gamma$ & $\kappa$ & $\nu_{0}$ \\
\hline & & $\boldsymbol{\Theta}_{\text {in }}$ & 0.6005 & -0.6815 & 0.4867 & 2.02 & 0.4961 \\
$J_{\mathcal{N}}(\boldsymbol{\Theta})$ & $\mathbb{E}_{\mathcal{N}}^{\mathrm{Am}}$ & $\boldsymbol{\Theta}^{\star}$ & 0.5953 & -0.7210 & 0.0527 & 3.3615 & 0.0584 \\
$J_{N}(\boldsymbol{\Theta})$ & $\mathbb{E}_{N}^{\mathrm{Am}}$ & $\boldsymbol{\Theta}^{\star}$ & 0.5144 & -0.7964 & 0.0521 & 2.5906 & 0.0554 \\
$\widetilde{J}_{\mathcal{N}}(\boldsymbol{\Theta})$ & $\mathbb{E}_{\mathcal{N}}^{\mathrm{Eu}}$ & $\boldsymbol{\Theta}^{\star}$ & 0.4095 & -0.6818 & 0.0516 & 1.6262 & 0.0567 \\
$\widetilde{J}_{\mathrm{CF}}(\boldsymbol{\Theta})$ & & $\boldsymbol{\Theta}^{\star}$ & 0.3927 & -0.6518 & 0.0580 & 1.4554 & 0.0546
\end{tabular}

TABLE 6 . Parameters obtained by the calibration on American put options given on the Google stock using different methods.

The results of the run-time performance of the different methods is given in Table 7. We observe that the detailed approach is much more cost-intense than the proposed surrogate models. The cost can be drastically reduced from a couple of days to less than an hour. Notably, we observe the substantial speed-up obtained by evaluating model prices with the closed-form solutions. This approach appears to us the most efficient when dealing with European options. However, taking into consideration the additional time for preprocessing the data in the DAS, the total time for calibration with this method can be much slower than the calibration with American options using the RBM, depending how 


\begin{tabular}{lccccc}
\hline Method & $\mathbb{E}(\boldsymbol{\mu})$ & \# iter. & $\# J$ & calib. time & pre-process. time for $P^{\text {obs }}$ \\
\hline$J_{\mathcal{N}}(\boldsymbol{\Theta})$ & $\mathbb{E}_{\mathcal{N}}^{\mathrm{Am}}$ & 35 & 219 & $68.72 \mathrm{hrs}$ & \\
$J_{N}(\boldsymbol{\Theta})$ & $\mathbb{E}_{N}^{\mathrm{Am}}$ & 38 & 260 & $44.20 \mathrm{~min}$ & \\
$\widetilde{J}_{\mathcal{N}}(\boldsymbol{\Theta})$ & $\mathbb{E}_{\mathcal{N}}^{\mathrm{Eu}}$ & 34 & 207 & $56.47 \mathrm{~min}$ & $4.96 \mathrm{hrs}$ \\
$\widetilde{J}_{\mathrm{CF}}(\boldsymbol{\Theta})$ & & 43 & 265 & $4.30 \mathrm{~min}$ & $4.96 \mathrm{hrs}$ \\
\hline
\end{tabular}

TABLE 7. Computational time for calibrating American put options given on the Google stock in the Heston model using different methods.

often the calibration has to be performed with new market data. However, the DAS preprocessing time could be sped up by implementing more advanced tree methods. We also note that, in contrast to the DAS, the RBM approach allows us to control the accuracy by increasing the dimension of the reduced spaces.
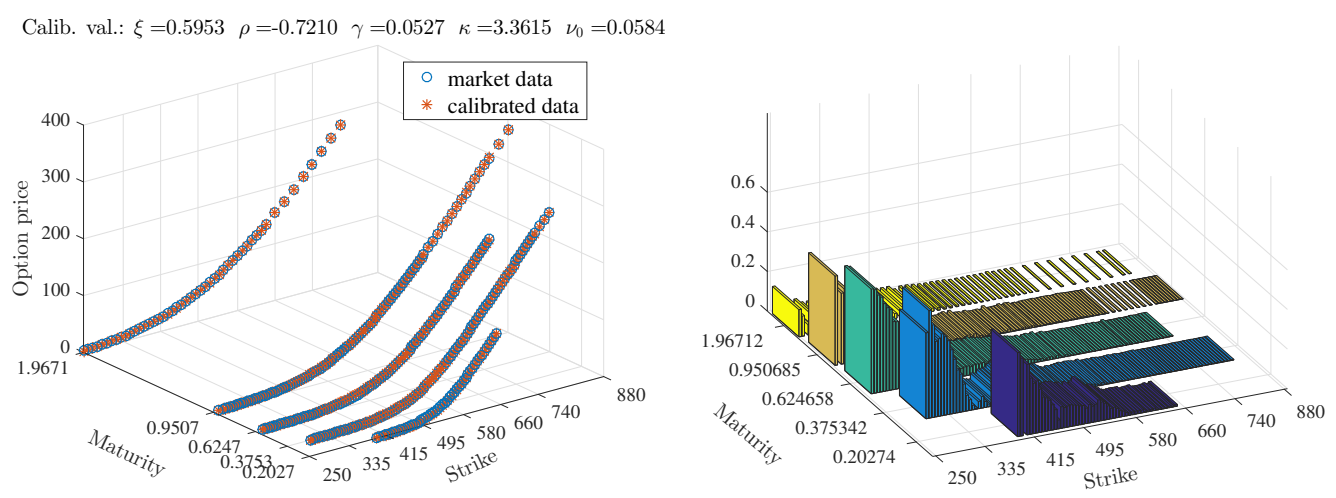

Detailed problem, $J_{\mathcal{N}}(\boldsymbol{\Theta})$
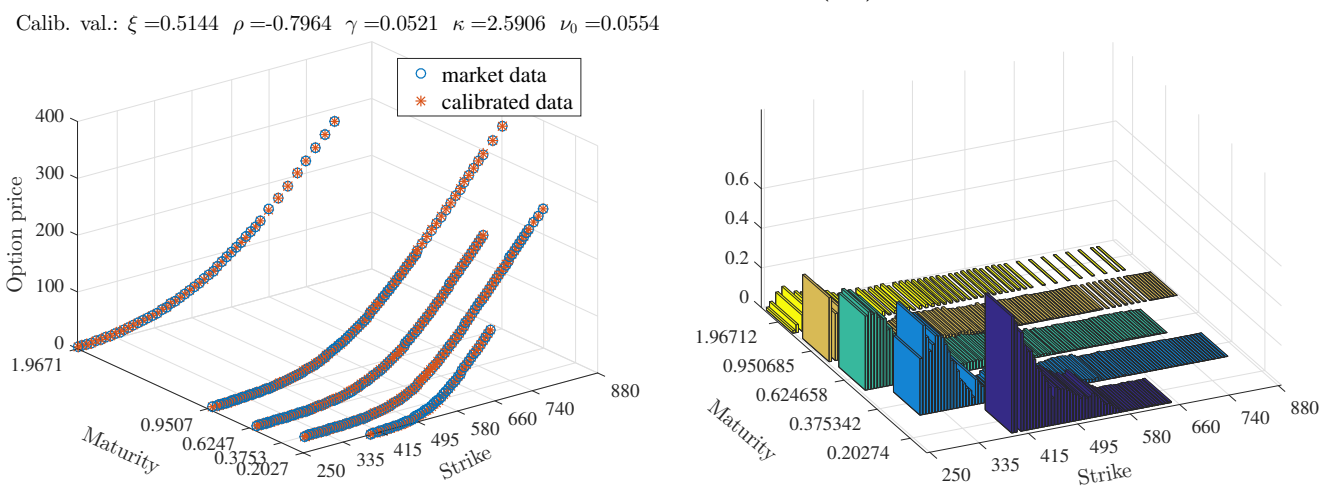

Reduced problem, $J_{N}(\boldsymbol{\Theta})$

Figure 5. Left: the Google data set of American put options (circles) and the calibrated model data in the Heston model (stars). Right: the relative error of the market and calibrated data, $\left|P_{i}^{\text {obs }}-P_{i}^{s, \operatorname{Am}}\left(\Theta^{\star}\right)\right| / P_{i}^{\text {obs }}$, $i=1, \ldots, M, s=\{\mathcal{N}, N\}$.

Figure 5 shows the calibration results based on market data for the detailed and the RBM approaches and the results for the two DAS are provided in Figure 6. The relative error for all approaches does not exceed $50 \%$ and increases in the out-of-the money region, 
which corresponds to the smallest option prices. To reduce this effect, one could consider different weights in the objective functional, e.g., imposing larger weights for small option price values.
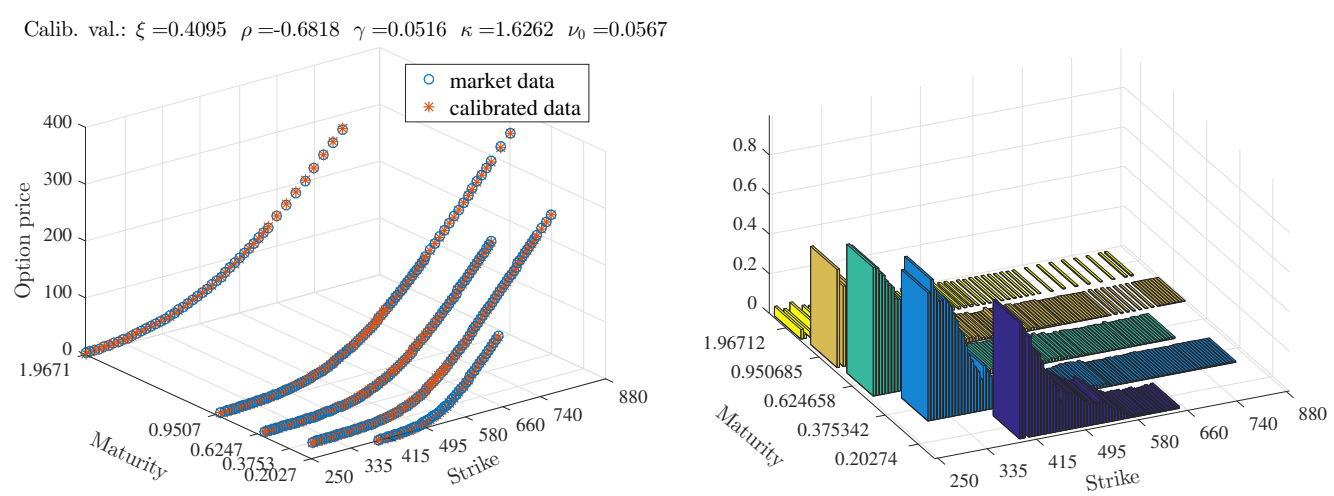

De-Americanized problem, $\widetilde{J}_{\mathcal{N}}(\boldsymbol{\Theta})$

Calib. val.: $\xi=0.3927 \quad \rho=-0.6518 \quad \gamma=0.0580 \quad \kappa=1.4554 \quad \nu_{0}=0.0546$
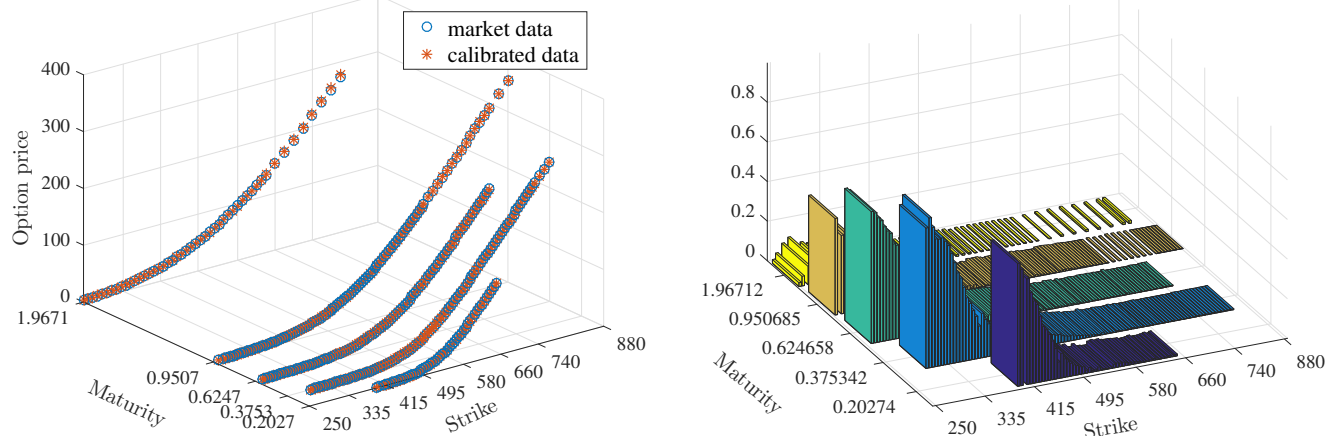

De-Americanized problem with closed-form solutions, $\widetilde{J}_{\mathrm{CF}}(\boldsymbol{\Theta})$

Figure 6. Left: the Google data set of the de-Americanized American put options (circles) and the calibrated model data in the Heston model (stars). Right: the relative error of the market and calibrated data, $\mid P_{i}^{\text {obs }}-$ $P_{i}^{s, \mathrm{Eu}}\left(\boldsymbol{\Theta}^{\star}\right) \mid / P_{i}^{\mathrm{obs}}, i=1, \ldots, M, s=\{\mathcal{N}, \mathrm{CF}\}$.

\section{CONCLUSion}

In this paper, we applied the reduced basis methodology to the calibration of European and American put options. In the case of American options we additionally considered a de-Americanization strategy. Both reduction strategies are compared numerically. While RBM techniques aim to achieve smaller dimensions for the discrete spaces in the variational formulations of the problem, the DAS replaces the constrained PDE model of an American option with the unconstrained model of a European option. By doing so, a model error of fixed size occurs, but the RBM offer flexibility by allowing us to adaptively adjust the accuracy. 


\section{REFERENCES}

Achdou, Y. (2005). An inverse problem for a parabolic variational inequality arising in volatility calibration with American options. SIAM J. Control Optim., 43(5):1583-1615 (electronic), doi:10.1137/S0363012903424423.

Achdou, Y. and Pironneau, O. (2005a). Computational methods for option pricing, volume 30 of Frontiers in Applied Mathematics. Society for Industrial and Applied Mathematics (SIAM), Philadelphia, PA.

Achdou, Y. and Pironneau, O. (2005b). Numerical procedure for calibration of volatility with American options. Applied Mathematical Finance, 12(3):201-241, doi:10.1080/1350486042000297252.

Balajewicz, M., Amsallem, D., and Farhat, C. (2016). Projection-based model reduction for contact problems. International Journal for Numerical Methods in Engineering, 106(8):644-663, doi:10.1002/nme.5135.

Balajewicz, M. and Toivanen, J. (2016). Reduced order models for pricing American options under stochastic volatility and jump-diffusion models. Procedia Computer Science, 80:734-743, doi:10.1016/j.procs.2016.05.360. International Conference on Computational Science 2016, ICCS 2016, 6-8 June 2016, San Diego, California, USA.

Barrault, M., Maday, Y., Nguyen, N. C., and Patera, A. T. (2004). An 'empirical interpolation' method: application to efficient reduced-basis discretization of partial differential equations. Comptes Rendus Mathematique, 339(9):667 - 672, doi:10.1016/j.crma.2004.08.006.

Black, F. and Scholes, M. (1973). The pricing of options and corporate liabilities. J. Polit. Econ., 81(3):637-654, doi:10.1086/260062.

Bouchouev, I. and Isakov, V. (1997). The inverse problem of option pricing. Inverse Problems, 13(5):L11-L17, doi:10.1088/0266-5611/13/5/001.

Brezzi, F., Hager, W. W., and Raviart, P.-A. (1978). Error estimates for the finite element solution of variational inequalities. II. Mixed methods. Numer. Math., 31(1):1-16, doi:10.1007/BF01396010.

Broadie, M. and Glasserman, P. (1997). Pricing American-style securities using simulation. J. Econom. Dynam. Control, 21(8-9):1323-1352, doi:10.1016/S0165-1889(97)00029-8.

Burkovska, O. (2016). Reduced Basis Methods for Option Pricing and Calibration. PhD thesis, Technische Universität München.

Burkovska, O., Gass, M., Glau, K., Mahlstedt, M., Schoutens, W., and Wohlmuth, B. (2018). Calibration to American options: numerical investigation of the de-Americanization method. Quantitative Finance, 0(0):1-23, doi:10.1080/14697688.2017.1417622.

Burkovska, O., Haasdonk, B., Salomon, J., and Wohlmuth, B. (2015). Reduced basis methods for pricing options with the Black-Scholes and Heston models. SIAM J. Financial Math., 6(1):685-712, doi:10.1137/140981216.

Carr, P. and Wu, L. (2010). Stock options and credit default swaps: A joint framework for valuation and estimation. Journal of Financial Econometrics, 8(4):409-449, doi:10.1093/jjfinec/nbp010.

CBOE (2009). The CBOE volatility index - VIX. CBOE.

Clarke, N. and Parrott, K. (1999). Multigrid for American option pricing with stochastic volatility. Applied Mathematical Finance, 6(3):177-195, doi:10.1080/135048699334528.

Cont, R., Lantos, N., and Pironneau, O. (2011). A reduced basis for option pricing. SIAM J. Financial Math., 2(1):287-316, doi:10.1137/10079851X. 
Cox, J. C., Ross, S. A., and Rubinstein, M. (1979). Option pricing: A simplified approach. Journal of Financial Economics, 7(3):229-263, doi:10.1016/0304-405X(79)90015-1.

Düring, B. and Fournié, M. (2012). High-order compact finite difference scheme for option pricing in stochastic volatility models. J. Comput. Appl. Math., 236(17):4462-4473, doi:10.1016/j.cam.2012.04.017.

Egger, H. and Engl, H. W. (2005). Tikhonov regularization applied to the inverse problem of option pricing: convergence analysis and rates. Inverse Problems, 21(3):1027-1045, doi:10.1088/0266-5611/21/3/014.

Fang, F. and Oosterlee, C. (2011). A Fourier-Based Valuation Method for Bermudan and Barrier Options under Heston's Model. SIAM Journal on Financial Mathematics, 2(1):439-463, doi:10.1137/100794158.

Fu, M. C., Laprise, S. B., Madan, D. B., Su, Y., and Wu, R. (2001). Pricing American options: A comparison of Monte Carlo simulation approaches. Journal of Computational Finance, 4(3):39-88, doi:10.21314/JCF.2001.066.

Gerlich, F., Giese, A. M., Maruhn, J. H., and Sachs, E. W. (2012). Parameter identification in financial market models with a feasible point SQP algorithm. Comput. Optim. Appl., 51(3):1137-1161, doi:10.1007/s10589-010-9369-8.

Glas, S. and Urban, K. (2014). On noncoercive variational inequalities. SIAM J. Numer. Anal., 52(5):2250-2271, doi:10.1137/130925438.

Glowinski, R., Lions, J.-L., and Trémolières, R. (1981). Numerical analysis of variational inequalities, volume 8 of Studies in Mathematics and its Applications. North-Holland Publishing Co., Amsterdam-New York. Translated from the French.

Haasdonk, B. (2013). Convergence rates of the POD-greedy method. ESAIM Math. Model. Numer. Anal., 47(3):859-873, doi:10.1051/m2an/2012045.

Haasdonk, B. and Ohlberger, M. (2008). Reduced basis method for finite volume approximations of parametrized linear evolution equations. M2AN Math. Model. Numer. Anal., 42(2):277-302, doi:10.1051/m2an:2008001.

Haasdonk, B., Salomon, J., and Wohlmuth, B. (2012). A reduced basis method for parametrized variational inequalities. SIAM J. Numer. Anal., 50(5):2656-2676, doi:10.1137/110835372.

Hesthaven, J. S., Rozza, G., and Stamm, B. (2016). Certified reduced basis methods for parametrized partial differential equations. SpringerBriefs in Mathematics. Springer, Cham; BCAM Basque Center for Applied Mathematics, Bilbao.

Heston, S. L. (1993). A closed-form solution for options with stochastic volatility with applications to bond and currency options. The Review of Financial Studies, 6(2):32743, doi:10.1093/rfs/6.2.327.

Hilber, N., Reichmann, O., Schwab, C., and Winter, C. (2013). Computational methods for quantitative finance. Finite element methods for derivative pricing. Springer Finance.

Hull, J. C. (2003). Options, Futures, and Other Derivative Securities. Prentice-Hall, fifth edition.

Janek, A., Kluge, T., Weron, R., and Wystup, U. (2011). FX smile in the Heston model. In Statistical tools for finance and insurance, pages 133-162. Springer, Heidelberg.

Kikuchi, N. and Oden, J. T. (1988). Contact problems in elasticity: a study of variational inequalities and finite element methods, volume 8 of SIAM Studies in Applied Mathematics. Society for Industrial and Applied Mathematics (SIAM), Philadelphia, PA.

Kunoth, A., Schneider, C., and Wiechers, K. (2012). Multiscale methods for the valuation of American options with stochastic volatility. International Journal of Computer Mathematics, 89(9):1145-1163, doi:10.1080/00207160.2012.672732. 
Lee, D. D. and Seung, H. S. (1999). Learning the parts of objects by non-negative matrix factorization. Nature, 401(6755):788-791, doi:10.1038/44565.

Levendorskii, S. Z. (2004). Early exercise boundary and option prices in Lévy driven models. Quantitative Finance, 4(5):525-547, doi:10.1080/14697680400023295.

Longstaff, F. A. and Schwartz, E. S. (2001). Valuing American options by simulation: a simple least-squares approach. Review of Financial studies, 14(1):113-147, doi:10.1093/rfs/14.1.113.

Mayerhofer, A. and Urban, K. (2016). A reduced basis method for parabolic partial differential equations with parameter functions and application to option pricing. Journal of Computational Finance, 20(2):71-106, doi:10.21314/JCF.2016.323.

Mrázek, M., Pospíšil, J., and Sobotka, T. (2014). On optimization techniques for calibration of stochastic volatility models. In AMCM 2015, November 28-30, 2014, Athens, Greece, pages $34-40$.

Peherstorfer, B., Gómez, P., and Bungartz, H.-J. (2015). Reduced models for sparse grid discretizations of the multi-asset Black-Scholes equation. Advances in Computational Mathematics, 41(5):1365-1389, doi:10.1007/s10444-015-9421-4.

Pironneau, O. (2009). Calibration of options on a reduced basis. J. Comput. Appl. Math., 232(1):139-147, doi:10.1016/j.cam.2008.10.070.

Pironneau, O. (2011). Reduced basis for vanilla and basket options. Risk and Decision Analysis, 2(4):185-194, doi:10.3233/RDA-2011-0045.

Pironneau, O. (2012). Proper orthogonal decomposition for pricing options. Journal of Computational Finance, 16(1):33-46, doi:10.21314/JCF.2012.246.

Quarteroni, A., Manzoni, A., and Negri, F. (2016). Reduced basis methods for partial differential equations, volume 92 of Unitext. Springer, Cham.

Quarteroni, A. and Valli, A. (1994). Numerical approximation of partial differential equations, volume 23 of Springer Series in Computational Mathematics. Springer-Verlag, Berlin.

Rogers, L. C. G. (2002). Monte Carlo valuation of American options. Math. Finance, 12(3):271-286, doi:10.1111/1467-9965.02010.

Rovas, D. (2003). Reduced-basis output bound methods for parametrized partial differential equations. $\mathrm{PhD}$ thesis, Massachusetts Institute of Technology.

Rozza, G. and Veroy, K. (2007). On the stability of the reduced basis method for Stokes equations in parametrized domains. Comput. Methods Appl. Mech. Engrg., 196(7):12441260, doi:10.1016/j.cma.2006.09.005.

Rubinstein, M. (1994). Implied binomial trees. The Journal of Finance, 49(3):771-818, doi:10.2307/2329207.

Sachs, E. W. and Schneider, M. (2014). Reduced-order models for the implied variance under local volatility. Int. J. Theor. Appl. Finance, 17(8):1450053, 23, doi:10.1142/S0219024914500538.

Schoutens, W., Simons, E., and Tistaert, J. (2004). A perfect calibration! Now what? Wilmott Magazine, pages 66-78.

Winkler, G., Apel, T., and Wystup, U. (2001). Valuation of options in Heston's stochastic volatility model using finite element methods. In Foreign Exchange Risk, pages 283-303. Risk Publications, London.

Wohlmuth, B. I. (2000). A mortar finite element method using dual spaces for the Lagrange multiplier. SIAM J. Numer. Anal., 38(3):989-1012, doi:10.1137/S0036142999350929.

Zhang, Z., Bader, E., and Veroy, K. (2016). A slack approach to reduced-basis approximation and error estimation for variational inequalities. C. R. Math. Acad. Sci. Paris, 

354(3):283-289, doi:10.1016/j.crma.2015.10.024. 


\section{Appendices}

Appendix A. Google market data

\begin{tabular}{|c|c|c|c|c|c|c|c|c|c|c|c|}
\hline$K \backslash T$ & 0.2027 & 0.3753 & 0.6247 & 0.9507 & 1.9671 & $K \backslash T$ & 0.2027 & 0.3753 & 0.6247 & 0.9507 & 1.9671 \\
\hline 250 & & & & & 2.50 & $\overline{540}$ & 30.20 & 36.40 & 44.45 & 52.70 & 72.65 \\
\hline 260 & & & & 1.20 & 2.90 & 545 & 33.25 & 39.25 & 47.60 & 56.10 & \\
\hline 265 & & & 0.55 & & & 550 & 37.15 & 42.30 & 50.25 & 58.95 & 78.50 \\
\hline 270 & & & 0.62 & 1.30 & 3.30 & 555 & 40.80 & 45.45 & 53.15 & 61.85 & \\
\hline 275 & & & 0.68 & 1.50 & & 560 & 44.30 & 49.00 & 56.55 & 64.80 & 84.05 \\
\hline 280 & & & 0.75 & 1.62 & 3.80 & 565 & 48.25 & 52.35 & 59.40 & 67.50 & \\
\hline 285 & & & 0.80 & 1.73 & & 570 & 51.95 & 56.00 & 63.50 & 71.10 & 90.40 \\
\hline 290 & & & 0.88 & 1.80 & 4.65 & 575 & 55.90 & 59.80 & 66.70 & 73.90 & \\
\hline 295 & & & 0.97 & 1.95 & & 580 & 60.20 & 63.70 & 69.85 & 77.25 & 96.45 \\
\hline 300 & & 0.28 & 1.00 & 2.10 & 5.35 & 585 & 64.50 & 67.10 & 74.25 & 81.30 & \\
\hline 305 & & 0.40 & 1.12 & 2.27 & & 590 & 68.80 & 71.60 & 77.85 & 84.45 & 103.00 \\
\hline 310 & & 0.40 & 1.20 & 2.45 & 6.25 & 595 & 73.35 & 76.30 & 81.70 & 88.20 & \\
\hline 315 & & 0.40 & 1.30 & 2.67 & & 600 & 77.35 & 80.65 & 85.10 & 91.55 & 108.85 \\
\hline 320 & & 0.47 & 1.38 & 2.85 & 7.40 & 605 & 82.35 & 84.80 & 89.65 & 95.20 & \\
\hline 325 & & 0.57 & 1.50 & 3.12 & & 610 & 87.30 & 89.65 & 93.20 & 99.10 & 116.45 \\
\hline 330 & & 0.65 & 1.60 & 3.40 & 8.10 & 615 & 92.50 & 94.05 & 97.40 & 102.90 & \\
\hline 335 & & 0.72 & 1.75 & 3.60 & & 620 & 96.65 & 98.45 & 101.85 & 106.80 & 123.75 \\
\hline 340 & & 0.78 & 1.90 & 3.85 & 9.65 & 625 & 101.30 & 103.00 & 106.40 & 111.80 & \\
\hline 345 & & 0.82 & 2.05 & 4.20 & & 630 & 106.50 & 107.25 & 110.60 & 116.00 & 130.65 \\
\hline 350 & & 0.88 & 2.23 & 4.55 & 10.90 & 635 & 111.75 & 112.95 & 115.00 & 120.25 & \\
\hline 355 & & 0.97 & 2.40 & 4.90 & & 640 & 117.20 & 117.25 & 119.85 & 124.40 & 138.10 \\
\hline 360 & & 1.05 & 2.70 & 5.30 & 12.55 & 645 & 121.45 & 122.30 & 124.40 & 128.55 & \\
\hline 365 & & 1.12 & 2.90 & 5.65 & & 650 & 127.25 & 126.70 & 128.90 & 132.55 & \\
\hline 370 & & 1.25 & 3.17 & 6.15 & 14.30 & 655 & & 131.45 & 133.70 & 137.15 & \\
\hline 375 & & 1.38 & 3.45 & 6.65 & & 660 & & 136.45 & 138.15 & 141.80 & 153.70 \\
\hline 380 & & 1.52 & 3.75 & 7.15 & 16.45 & 665 & & 141.45 & 143.05 & 146.10 & \\
\hline 385 & & 1.65 & 4.10 & 7.65 & & 670 & & 146.65 & 147.35 & 150.50 & \\
\hline 390 & & 2.05 & 4.45 & 8.15 & 18.30 & 675 & & 151.80 & 152.05 & 154.90 & \\
\hline 395 & 0.93 & 2.25 & 4.85 & 8.80 & & 680 & & 156.20 & 158.25 & 159.45 & 169.45 \\
\hline 400 & 1.05 & 2.50 & 5.30 & 9.45 & 20.85 & 685 & & 161.20 & 161.60 & 164.05 & \\
\hline 405 & & 2.73 & 5.75 & 10.10 & & 690 & & 166.30 & 166.55 & 168.65 & \\
\hline 410 & 1.40 & 3.08 & 6.30 & 10.75 & 22.85 & 695 & & 171.30 & 171.45 & 173.15 & \\
\hline 415 & 1.48 & 3.35 & 6.85 & 11.55 & & 700 & & 176.20 & 176.45 & 178.15 & 186.40 \\
\hline 420 & 1.65 & 3.75 & 7.45 & 12.40 & 25.55 & 705 & & 181.10 & 181.75 & & \\
\hline 425 & 1.93 & 4.10 & 8.15 & 13.40 & & 710 & & 186.15 & 186.25 & 188.40 & \\
\hline 430 & 2.10 & 4.55 & 8.80 & 14.20 & 28.30 & 715 & & 191.10 & 191.35 & & \\
\hline 435 & 2.33 & 5.05 & 9.55 & 15.35 & & 720 & & 196.05 & 195.85 & 197.15 & 203.70 \\
\hline 440 & 2.70 & 5.55 & 10.40 & 16.25 & 31.35 & 725 & & 201.05 & 201.80 & & \\
\hline 445 & 3.10 & 6.10 & 11.25 & 17.35 & & 730 & & 205.85 & 206.50 & 206.95 & \\
\hline 450 & 3.50 & 6.80 & 12.30 & 18.65 & 34.00 & 735 & & 211.00 & 211.05 & & \\
\hline 455 & 3.92 & 7.50 & 13.25 & 19.80 & & 740 & & 216.80 & 216.80 & 216.20 & 222.30 \\
\hline 460 & 4.25 & 8.35 & 14.25 & 21.20 & 37.80 & 745 & & 221.80 & 221.90 & & \\
\hline 465 & 5.20 & 9.20 & 15.45 & 22.60 & & 750 & & 226.55 & 226.85 & 226.20 & \\
\hline 470 & 5.85 & 10.20 & 16.70 & 23.90 & 41.45 & 755 & & 231.80 & & & \\
\hline 475 & 6.65 & 11.25 & 18.00 & 25.30 & & 760 & & 236.80 & & 236.15 & 240.60 \\
\hline 480 & 7.60 & 12.40 & 19.45 & 27.15 & 45.20 & 765 & & 241.95 & & & \\
\hline 485 & 8.65 & 13.70 & 20.95 & 28.55 & & 770 & & 246.95 & & 246.05 & \\
\hline 490 & 9.85 & 15.10 & 22.55 & 30.75 & 49.20 & 775 & & 251.80 & & & \\
\hline 495 & 11.15 & 16.55 & 24.25 & 32.60 & & 780 & & 256.80 & & 256.05 & 258.90 \\
\hline 500 & 12.65 & 18.25 & 26.00 & 34.60 & 53.50 & 790 & & 267.00 & & 265.70 & \\
\hline 505 & 14.05 & 20.00 & 27.90 & 36.80 & & 800 & & 276.95 & & 276.05 & 277.70 \\
\hline 510 & 16.05 & 21.95 & 30.00 & 38.70 & 58.15 & 810 & & 287.25 & & 286.05 & \\
\hline 515 & 17.75 & 23.90 & 32.15 & 41.45 & & 820 & & & & 296.05 & \\
\hline 520 & 19.90 & 26.20 & 34.40 & 43.65 & 62.90 & 830 & & & & 306.05 & \\
\hline 525 & 22.50 & 28.55 & 36.70 & 46.05 & & 840 & & & & 316.05 & \\
\hline 530 & 24.70 & 31.05 & 39.20 & 48.55 & 67.90 & 860 & & & & 336.05 & \\
\hline 535 & 27.40 & 33.50 & 41.85 & 50.85 & & 880 & & & & 356.05 & \\
\hline
\end{tabular}

TABLE A.1. Google market data consisting of 401 American put options with $S_{0}=523,755$ on February 2nd, 2015.

Olena Burkovska

Department of Scientific Computing, Florida State University, 400 Dirac Science Library, TAllahassee, FL 32306-4120, USA 
E-mail address: oburkovska@fsu.edu

\section{Kathrin Glau}

Queen Mary University of London, School of Mathematical Sciences, Mile End Road, E1 4NS, LONDON, UK;

Technische Universität München, Chair of Mathematical Finance, Parkring 11, 85748 Garching B. München, Germany

E-mail address: k.glau@qmul.ac.uk; kathrin.glau@tum.de

Mirco Mahlstedt

Chair of Mathematical Finance, Technische Universität München, Parkring 11, 85748

Garching B. München, Germany

E-mail address: mirco.mahlstedt@me.com

BARBARA WOHLMUTH

Institute for Numerical Mathematics (M2), Technische Universität München, Boltzmannstrasse 3, 85748 Garching B. München, Germany

E-mail address: wohlmuth@ma.tum.de 